\title{
Modeling and Simulation of a Ternary System for Macromolecular Microsphere Composite Hydrogels
}

\author{
Guanghua Ji, Yuqi Yang* and Hui Zhang \\ Laboratory of Mathematics and Complex Systems (Ministry of Education), School \\ of Mathematical Sciences, Beijing Normal University, Beijing 100875, P.R. China.
}

Received 10 May 2020; Accepted (in revised version) 4 August 2020.

\begin{abstract}
In this paper, we study a ternary system for macromolecular microsphere composite (MMC) hydrogels. Assuming that the graft chains are distributed randomly around the macromolecular microspheres, a phase transition model was constructed. The stabilised-scalar auxiliary variable (S-SAV) approach is used to present a first-order energy stable scheme for solving the nonlinear system. Some numerical experiments are carried out to show the accuracy of the scheme, including the mass conservation of the volume fractions, the decrease in the modified energy, and the influence of different parameters.
\end{abstract}

AMS subject classifications: 65H10, 65M06, 65M22

Key words: MMC hydrogels, ternary system, unconditionally energy stable, S-SAV.

\section{Introduction}

The macromolecular microsphere composite (MMC) hydrogel, proposed by Huang et al. [25] in 2010, is a new type of hydrogel. Later on, various materials have been used to synthesise the MMC hydrogel in a similar way $[21,23,40,45,66]$. The formation process is described as follows: evenly add monomers to the irradiated macromolecular microsphere (MMS) emulsion, and heat the mixture. The monomers will chemically graft onto the surface of the MMSs and grow into grafted polymer chains. It is possible for a polymer chain to graft onto another MMS or another polymer chain, so the MMSs can be joined by polymer chains. If the length of the polymer chain becomes larger than the distance between two MMSs, the chain entangles. The mixture finally transforms into a solid gel, the microstructure of which is shown in Fig. 1. Because of the chemical grafting and the entangled polymer chains, the MMC hydrogel has a well-defined network structure and very high mechanical strength $[26,46]$. This led to wide application of MMC hydrogels in biomedical and industrial areas $[21,23,25,40,66]$. However, here we mainly focus on the method for simulating the phase transition of MMC hydrogels.

*Corresponding author. Email addresses: ghji@bnu.edu.cn (G. Ji), 201721130050@mail.bnu.edu.cn (Y. Yang), hzhang@bnu.edu.cn (H. Zhang) 


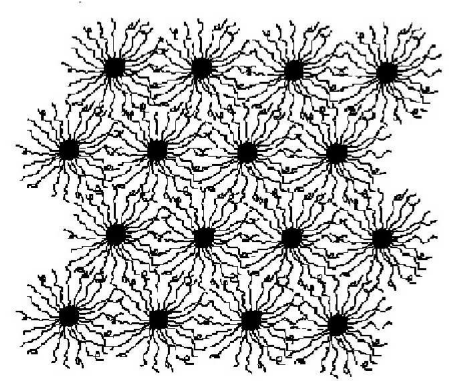

Figure 1: The microstructure of the MMC hydrogel.

The time-dependent Ginzburg-Landau (TDGL) mesoscale simulation method is based on the Cahn-Hilliard-Cook nonlinear diffusion equation and is used in the modelling of the phase transition of the polymer blends [19]. The method connects the rate of change of one or more spatiotemporal order parameters in time with the derivatives of a free energy, which is a functional of these order parameters. In particular, for incompressible binary polymer blends, the following equation is used:

$$
\frac{\partial \phi(\mathbf{x}, t)}{\partial t}=\nabla \cdot D \nabla \frac{\delta F[\phi(\mathbf{x}, t)]}{\delta \phi(\mathbf{x}, t)}
$$

where $\phi$ is the spatiotemporal order parameter, $D$ the mobility, and

$$
F[\phi]=\int d \mathbf{r}\left\{f[\phi]+\kappa(\phi)|\nabla \phi|^{2}\right\}
$$

the Ginzburg-Landau type free energy with the variable interface coefficient $\kappa(\phi)$ and the Flory-Huggins free energy of mixing $f[\phi]$.

In recent years, the TDGL method has been used to simulate the phase transitions of MMC hydrogels in binary systems. Zhai et al. [61] introduced a reticular free energy to describe the network structure of MMC hydrogels, derived a binary MMC-TDGL equation, and used a spectral method for its solution. Li et al. $[29,30]$ solved the binary MMC-TDGL equation by a semi-implicit difference scheme and employed a finite difference method in combination with a convex splitting technique for the energy functional. Xu et al. [51] presented a stabilised semi-implicit scheme for the binary MMC-TDGL equation. Yang [53] employed the invariant energy quadratisation (IEQ) method to the phase field model for homopolymer blends (PF-HB). Thus the TDGL method is suitable for simulating the phase transition of MMC hydrogels in a binary system. Nevertheless, there are only a few works investigating the corresponding methods for ternary systems.

In binary systems, an important assumption is that the number of graft chains around an MMS is proportional to the perimeter $[29,30,51,61]$. That is, the volume fraction of the polymer chains is proportional to the volume fraction of the MMSs. We can view MMSs and polymer chains as one component and water as the other. 
In this paper, we study more practical systems where graft chains are randomly distributed around MMSs - i.e. polymer chains, MMSs and water are viewed as the components of a ternary phase transition model of an MMC hydrogel.

To simulate the phase transition of the MMC hydrogel in a ternary system, we have two main tasks. First, using the three-component Cahn-Hilliard equations combined with the Ginzburg-Landau free energy, we develop ternary MMC-TDGL equations. In order to describe the reticular structure of the MMC hydrogel, we replace the Flory-Huggins energy with the reticular energy, and the reticular energy is calculated similar to [61]. Second, we solve the ternary MMC-TDGL equations. This is the main challenge because two coupled fourth-order nonlinear parabolic partial differential equations have to be solved.

There are various approaches to nonlinear equations. Usually, one writes the free energy functional as a sum of linear and nonlinear terms - viz.

$$
F[\phi]=\frac{1}{2} \int \phi \mathscr{L} \phi d \mathbf{x}+F_{1}[\phi],
$$

where $\mathscr{L}$ is a symmetric non-negative linear operator and $F_{1}$ a nonlinear term with lowerorder derivatives than $\mathscr{L}$. Besides, in the binary system, $\phi$ is a scalar but it is a vector in the ternary system. The linear term is usually treated implicitly, so the key is to figure out how to deal with the nonlinear term, since different approaches treat the nonlinear term differently. We now provide a brief introduction to numerical methods for three-component Cahn-Hilliard equations.

The convex splitting method was introduced in $[15,16]$. In this method the free energy functional is represented as the difference of two convex functionals - i.e. $F=F_{c}-F_{e}$, where both $F_{c}$ and $F_{e}$ are convex. The functional $F_{c}$ is treated implicitly, and $F_{e}$ explicitly. One can easily show that the convex splitting scheme unconditionally satisfies the discrete energy law. However, the nonlinear equations have to be solved at each time step since, usually, the implicit term $F_{c}$ is nonlinear - cf. Refs. [1, 2, 6, 7, 20, 24, 33, 42, 43, 47].

In the stabilisation method, the nonlinear term is treated explicitly and in order to avoid strict time step constraints, a linear stabiliser is added [18,28, 37, 38,69]. This is an efficient method since, at any time step, only linear equations have to be solved. However, the proof of the unconditional energy stability requires the boundedness of the secondorder derivative of the energy functional. In many cases, this condition is too strong, so that the energy functional is usually truncated to satisfy it $[4,32]$.

The invariant energy quadratisation (IEQ) method, proposed by Yang [53], has been applied to different phase field models $[5,12,20,49,54-60,67]$. In this approach, all the nonlinear terms are treated implicitly and the free energy has to be bounded from below. Although for the three-component Cahn-Hilliard equations a linear scheme can be constructed, it leads to a coupled system with variable coefficients changing at each time step. The modified energy is unconditionally stable, but the stability of the initial energy cannot be proven.

The scalar auxiliary variable (SAV) approach was proposed by Shen et al. [36] and later on Shen and $\mathrm{Hu}$ [35] considered higher-order SAV schemes. The nonlinear term is treated implicitly and it has to be bounded from below. A linear unconditionally energy stable 
scheme can be easily constructed and the equations are decoupled. Nevertheless, only the modified energy is unconditionally stable but not the original one. More applications can be found in [27,68] and the convergence and error analysis are presented in [31,34, 44].

A new Lagrange multiplier method has been recently proposed by Cheng et al. [11]. The nonlinear term is treated explicitly, but an implicit scalar auxiliary function is added. The method does not require the boundedness of the corresponding free energy functional or the nonlinear term from below and the original energy is unconditionally stable. However, for the three-component Cahn-Hilliard equations, four linear and two nonlinear equations have to be solved at each time step.

Recently, stabilisation terms are added to other numerical schemes in order to enhance their stability, so that large time steps are allowed while keeping the required accuracy. A Crank-Nicolson type scheme combined with convex splitting and stabilisation is used in $[41,48]$. The second-order backward differentiation formula (BDF) finite difference schemes in $[8-10,17,52]$ are also based on a convex splitting and stabilisation. For the anisotropic Cahn-Hilliard equation, the stabilisation terms used in IEQ approach [50] and SAV approach [3] aim to suppress non-physical spatial oscillations caused by the strong anisotropy. Besides, the stabilised-IEQ (S-IEQ) approach is considered in $[62,63]$ and stabilised-SAV (S-SAV) approach in $[64,65]$.

We used the SAV approach to simulate the ternary phase transition model of an MMC hydrogel. However, it leads to blow-up since the higher derivatives in the free energy functional are nonlinear and have to be treated explicitly. Therefore, a stabilisation term is added to the scheme i.e. an S-SAV approach is used.

The rest of the paper is organised as follows. In Section 2, we introduce a phase transition model for the ternary MMC hydrogels. In Section 3, a linear, first-order, unconditionally energy stable scheme is used to solve the nonlinear system from Section 2. In Section 4, we show the results of numerical simulations. Our conclusions and further discussion are in Section 5.

\section{A Phase Transition Model For MMC Hydrogels}

We consider a system consisting of MMSs, polymer chains and water. At the beginning of the synthesis, MMSs and monomers distribute evenly in water, so the initial volume fraction of MMSs, polymer chains and water, respectively, denoted by $\phi_{m}, \phi_{p}$ and $\phi_{w}$, are uniformly distributed in space. To construct a ternary phase transition model, we first establish TDGL equations in the ternary system.

\subsection{TDGL method}

The time-dependent Ginzburg-Landau (TDGL) mesoscale simulation method is widely used for simulating phase transitions in multicomponent polymer blends. For an $n$-component system, the method begins with a continuity equation for each component $i$ of the 
mixture [19], i.e.

$$
\frac{\partial \phi_{i}(\mathbf{x}, t)}{\partial t}=-\nabla \cdot j_{i}(\mathbf{x}, t)
$$

where $\phi_{i}(\mathbf{x}, t)$ is the spatiotemporal concentration of component $i$ such that

$$
\phi_{1}+\phi_{2}+\cdots+\phi_{n}=1
$$

and $j_{i}(\mathbf{x}, t)$ is the mass current of the component $i$ related to the chemical potential $\mu_{j}$ by the Fick's law

$$
j_{i}(\mathbf{x}, t)=-\sum_{j=1}^{n-1} D_{i j} \nabla \mu_{j}
$$

with $D_{i j}$ denoting the mobility of component $i$ due to $j$. The chemical potential $\mu_{j}$ is thermodynamically related to the free energy functional $F\left[\phi_{1}(\mathbf{x}, t), \phi_{2}(\mathbf{x}, t), \ldots, \phi_{n-1}(\mathbf{x}, t)\right]$ by $\mu_{j}=\delta F / \delta \phi_{j}$.

Since the MMC hydrogel is composed of MMSs, polymer chains and water, we can describe the structure of the ternary system by the following TDGL equations:

$$
\begin{aligned}
& \frac{\partial \phi_{1}(\mathbf{x}, t)}{\partial t}=\nabla \cdot\left(D_{1} \nabla \frac{\delta F\left[\phi_{1}(\mathbf{x}, t), \phi_{2}(\mathbf{x}, t)\right]}{\delta \phi_{1}(\mathbf{x}, t)}\right), \\
& \frac{\partial \phi_{2}(\mathbf{x}, t)}{\partial t}=\nabla \cdot\left(D_{2} \nabla \frac{\delta F\left[\phi_{1}(\mathbf{x}, t), \phi_{2}(\mathbf{x}, t)\right]}{\delta \phi_{2}(\mathbf{x}, t)}\right), \\
& \phi_{3}=1-\phi_{1}-\phi_{2} .
\end{aligned}
$$

We model the free energy functional in the form of the Ginzburg-Landau type energy functional, incorporating the interface gradient terms of de-Gennes

$$
\frac{F\left[\phi_{1}, \phi_{2}\right]}{k_{B} T}=\int d \mathbf{x}\left\{\frac{f\left[\phi_{1}, \phi_{2}\right]}{k_{B} T}+\frac{1}{36} \sum_{i=1}^{3} \frac{a_{i}^{2}}{\phi_{i}}\left|\nabla \phi_{i}\right|^{2}\right\},
$$

where $a_{i}$ is the statistical segment length of component, $f\left[\phi_{1}, \phi_{2}\right]$ the Flory-Huggins free energy of mixing, and $(1 / 36) \sum_{i=1}^{3}\left(a_{i}^{2} / \phi_{i}\right)\left|\nabla \phi_{i}\right|^{2}$ the energy of the interface $i$, cf. [13, 14, 39]. However, since the MMC hydrogel has a well-defined network structure, we have to the reticular free energy density $f\left[\phi_{1}, \phi_{2}\right]$ in order to replace the Flory-Huggins free energy of mixing.

\subsection{Reticular free energy}

The free energy density $f$ is related to the mixing enthalpy $H$, the temperature $T$, and the mixing entropy $S$ by $f=H-T S$. The definition of the variables used in the forthcoming calculations is given in Table 1 .

The mixing entropy $H$,

$$
H=-k_{B} T V\left(\chi_{m p} \phi_{m} \phi_{p}+\chi_{m w} \phi_{m} \phi_{w}+\chi_{p w} \phi_{p} \phi_{w}\right)
$$


Table 1: Definition of variables.

\begin{tabular}{||l|l||}
\hline Variables & Definitions \\
\hline$n_{m}$ & the number of the macromolecular microspheres (MMSs) \\
\hline$n_{p}$ & the number of the segments \\
\hline$n_{w}$ & the number of the solvent (water) molecules \\
\hline$M$ & the lattice number that one MMS occupies \\
\hline$N$ & the polymerization degree of the polymer chains \\
\hline$V$ & the total lattice number of the system occupies, $V=M n_{m}+n_{p}+n_{w}$ \\
\hline$\phi_{m}$ & the volume fraction of the MMS, $\phi_{m}=M n_{m} / V$ \\
\hline$\phi_{p}$ & the volume fraction of the polymer chains, $\phi_{p}=n_{p} / V$ \\
\hline$\phi_{w}$ & the volume fraction of the solvent (water) molecules, $\phi_{s}=n_{s} / V$ \\
\hline
\end{tabular}

represents the interaction between any two components and $\chi_{m p}, \chi_{m w}, \chi_{p w}$ are, respectively, the interaction parameters between MMS and polymer, MMS and water, polymer and water.

Then, the mixing entropy $S$ is calculated by using the Boltzmann entropy theorem, Flory-Huggins lattice theory and the statistical thermodynamic method [22]. The Boltzmann entropy theorem reads as follows.

Theorem 2.1. The mixing entropy $S$ and microscopic configuration number of the system $\Omega$ are relates as

$$
S=k_{B} \ln \Omega
$$

where $k_{B}$ is the Boltzmann constant.

Taking into account the Flory-Huggins lattice theory, we make the following assumptions for our model:

- Each solvent molecule occupies a lattice, and the solvent molecules can replace each other.

- Each MMS occupies M lattices.

- All the polymer chains have the same polymerization degree $N$, and each polymer molecule occupies $\mathrm{N}$ connected lattices. The polymer chains are completely flexible, and all the configurations have equivalent energy.

- Each MMS has the same properties and they are independent of each other, which is the same as the polymer chains.

- Both ends of all the polymer chains are grafted onto the MMS surfaces.

For convenience, we restrict ourselves to two dimensions. The three-dimensional case can be considered analogously. MMSs and polymer chains cannot be distributed randomly 
because polymer chains are distributed among the MMSs. Therefore, using the statistical thermodynamic method, we can obtain the following microscopic configuration numbers of MMSs, polymer chains, and water:

$$
\begin{aligned}
& \Omega_{m}=\left(\frac{V}{\pi\left(\sqrt{\frac{M}{\pi}}+\frac{N}{2}\right)^{2} n_{m}}\right)^{n_{m}}, \\
& \Omega_{p}=\left(\frac{V}{\left(2 \sqrt{\frac{M}{\pi}}+N\right) \frac{n_{p}}{N}}\right)^{n_{p} / N}, \\
& \Omega_{w}=\left(\frac{V}{n_{w}}\right)^{n_{w}} .
\end{aligned}
$$

The mixing entropy $S$ of the system can be obtained by the Boltzmann entropy theorem, so that

$$
S=-k_{B} V\left[\frac{\phi_{m}}{M} \ln \left(\frac{\alpha \phi_{m}}{M}\right)+\frac{\phi_{p}}{N} \ln \left(\frac{\beta \phi_{p}}{N}\right)+\phi_{w} \ln \phi_{w}\right],
$$

where

$$
\alpha=\pi\left(\sqrt{\frac{M}{\pi}}+\frac{N}{2}\right)^{2}, \quad \beta=2 \sqrt{\frac{M}{\pi}}+N .
$$

Replacing the subscripts $m, p, w$ with $1,2,3$, we write the reticular free energy density in the form

$$
\begin{aligned}
f=k_{B} T V\left[\frac{\phi_{1}}{M} \ln \left(\frac{\alpha \phi_{1}}{M}\right)+\frac{\phi_{2}}{N} \ln \left(\frac{\beta \phi_{2}}{N}\right)\right. & +\phi_{3} \ln \phi_{3}+\chi_{12} \phi_{1} \phi_{2} \\
& \left.+\chi_{13} \phi_{1} \phi_{3}+\chi_{23} \phi_{2} \phi_{3}\right] .
\end{aligned}
$$

\subsection{Ternary MMC-TDGL equations}

The above calculations lead to the following ternary MMC-TDGL equations with $(x, y) \in$ $\Omega=[0, L]^{2}$ :

$$
\begin{aligned}
& \frac{\partial \phi_{1}}{\partial t}=D_{1} \Delta \frac{\delta F}{\delta \phi_{1}}, \\
& \frac{\partial \phi_{2}}{\partial t}=D_{2} \Delta \frac{\delta F}{\delta \phi_{2}}, \\
& \phi_{3}=1-\phi_{1}-\phi_{2},
\end{aligned}
$$

where $\phi_{1}$ and $\phi_{2}$ are equipped with periodic boundary conditions. The Eqs. (2.3a)-(2.3c) have the following property. 
Theorem 2.2. In the ternary MMC-TDGL equations, the volume fractions $\phi_{i}, i=1,2$ are mass-conservative - i.e.,

$$
\int_{\Omega} \phi_{i}(x, y, t) d x d y=\int_{\Omega} \phi_{i 0}(x, y, t) d x d y \text { for all } t>0 .
$$

Proof. Integrating the Eqs. (2.3a) and (2.3b) over $\Omega$ and using the Gaussian formula yields

$$
\frac{d}{d t} \int_{\Omega} \phi_{i} d x d y=\int_{\Omega} \frac{\partial \phi_{i}}{\partial t} d x d y=D_{i} \int_{\Omega} \Delta \frac{\delta F}{\delta \phi_{i}} d x d y=D_{i} \int_{\Omega} \nabla \frac{\delta F}{\delta \phi_{i}} \cdot \mathbf{n} d S .
$$

Since $\delta F / \delta \phi_{i}$ is periodic with respect to $x$ and $y$, we obtain

$$
\frac{d}{d t} \int_{\Omega} \phi_{i} d x d y=0
$$

Substituting the reticular free energy density (2.2) into the Eq. (2.1) and using (2.3c), we write the free energy functional $F\left[\phi_{1}, \phi_{2}\right]$ as

$$
\begin{aligned}
F\left[\phi_{1}, \phi_{2}\right]=\int d \mathbf{x}\{ & \frac{\phi_{1}}{M} \ln \left(\frac{\alpha \phi_{1}}{M}\right)+\frac{\phi_{2}}{N} \ln \left(\frac{\beta \phi_{2}}{N}\right)+\left(1-\phi_{1}-\phi_{2}\right) \ln \left(1-\phi_{1}-\phi_{2}\right) \\
& +\chi_{12} \phi_{1} \phi_{2}+\chi_{13} \phi_{1}\left(1-\phi_{1}-\phi_{2}\right)+\chi_{23} \phi_{2}\left(1-\phi_{1}-\phi_{2}\right) \\
& \left.+\frac{a_{1}^{2}}{36 \phi_{1}}\left|\nabla \phi_{1}\right|^{2}+\frac{a_{2}^{2}}{36 \phi_{2}}\left|\nabla \phi_{2}\right|^{2}+\frac{a_{3}^{2}}{36\left(1-\phi_{1}-\phi_{2}\right)}\left|\nabla\left(1-\phi_{1}-\phi_{2}\right)\right|^{2}\right\} .
\end{aligned}
$$

Theorem 2.3. The energy functional $F\left[\phi_{1}, \phi_{2}\right]$ of the system (2.3a)-(2.3c) decreases with time - i.e.

$$
\frac{d F}{d t} \leq 0
$$

Proof. Multiplying (2.3a) and (2.3b), respectively, by $\partial \phi_{1} / \partial t$ and $\partial \phi_{2} / \partial t$, summing the resulting equations and integrating it over $\Omega$ gives

$$
\frac{d F}{d t}=\sum_{i=1}^{2} \int \frac{\delta F}{\delta \phi_{i}} \frac{\partial \phi_{i}}{\partial t} d \mathbf{x}=-\sum_{i=1}^{2} D_{i} \int\left|\nabla \frac{\delta F}{\delta \phi_{i}}\right|^{2} \leq 0
$$

Note that we used Green's formula and the boundary conditions.

\section{Numerical Scheme}

To obtain the solution of the MMC-TDGL equations (2.3a)-(2.3c), we have to solve two coupled fourth-order nonlinear parabolic partial differential equations. Thus, we use the scalar auxiliary variable (SAV) approach proposed by Shen $[35,36]$. It is an efficient 
approach when dealing with high-order nonlinear equations and with the equation decoupling. For this, we write the free energy functional as the sum

$$
F[\phi]=\frac{1}{2} \int \phi \mathscr{L} \phi d \mathbf{r}+F_{1}[\phi]
$$

where $\mathscr{L}$ is a symmetric non-negative linear operator and $F_{1}$ a nonlinear term. We deal with the linear term implicitly and with the nonlinear term explicitly. However, since the highest-order term in the free energy functional of the ternary MMC-TDGL equations is not linear, all terms in the free energy functional have to be treated explicitly. In our simulations, the fully explicit scheme produces divergent numerical solutions. Therefore, a linear stabiliser is added to the SAV approach. By using the stabilised-scalar auxiliary variable (S$\mathrm{SAV}$ ) approach, we obtain an efficient unconditional stable energy scheme for high-order nonlinear equations.

Introducing the scalar auxiliary variable $r(t)=\sqrt{F\left[\phi_{1}, \phi_{2}\right]}$, we write the system (2.3a)$(2.3 c)$ as

$$
\begin{aligned}
& \frac{\partial \phi_{i}}{\partial t}=D_{i} \Delta \mu_{i}, \\
& \mu_{i}=\frac{r}{\sqrt{F\left[\phi_{1}, \phi_{2}\right]}} U_{i}, \\
& r_{t}=\frac{1}{2 \sqrt{F\left[\phi_{1}, \phi_{2}\right]}} \int_{\Omega} \sum_{j=1}^{2} U_{j} \frac{\partial \phi_{j}}{\partial t} d \mathbf{x},
\end{aligned}
$$

where $U_{i}=\delta F / \delta \phi_{i}$. The energy dissipation law of the new system (3.1a)-(3.1c) is easily obtained. Let

$$
(f(\mathbf{x}), g(\mathbf{x})):=\int_{\Omega} f(\mathbf{x}) g(\mathbf{x}) d \mathbf{x}
$$

be the $L^{2}$ inner product of spatial functions $f(\mathbf{x})$ and $g(\mathbf{x})$.

Theorem 3.1. The energy of the system (3.1a)-(3.1c) is unconditionally stable - i.e.

$$
\frac{d F}{d t} \leq 0 .
$$

Proof. Considering the sum of the inner products of functions (3.1a) and (3.1b), we have

$$
\begin{aligned}
& \sum_{i=1}^{2}\left(\frac{\partial \phi_{i}}{\partial t}, \mu_{i}\right)=\sum_{i=1}^{2} D_{i}\left(\Delta \mu_{i}, \mu_{i}\right), \\
& \sum_{i=1}^{2}\left(\frac{\partial \phi_{i}}{\partial t}, \mu_{i}\right)=\sum_{i=1}^{2}\left(\frac{\partial \phi_{i}}{\partial t}, \frac{\delta F}{\delta \phi_{i}}\right) .
\end{aligned}
$$


The energy dissipation law can be now obtained by summing the above equations and eliminating $\sum_{i=1}^{2}\left(\partial \phi_{i} / \partial t, \mu_{i}\right)$ by using the periodicity of the boundary conditions, so that

$$
\frac{d F}{d t}=\sum_{i=1}^{2}\left(\frac{\delta F}{\delta \phi_{i}}, \frac{\partial \phi_{i}}{\partial t}\right)=\sum_{i=1}^{2} D_{i}\left(\Delta \mu_{i}, \mu_{i}\right) \leq 0 .
$$

Remark 3.1. Since the free energy functional $F\left[\phi_{1}, \phi_{2}\right]$ can be negative, we define the scalar auxiliary variable by $r(t)=\sqrt{F\left[\phi_{1}, \phi_{2}\right]+C}$, where $C \geq 0$ and $F\left[\phi_{1}, \phi_{2}\right] \geq-C$. Thus, the free energy functional $F\left[\phi_{1}, \phi_{2}\right]$ needs to be bounded from below.

\subsection{The first-order S-SAV scheme}

Given the initial value

$$
\begin{aligned}
& \phi_{1}(t=0)=\phi_{1}^{0}, \\
& \phi_{2}(t=0)=\phi_{2}^{0}, \\
& r(t=0)=\sqrt{F\left[\phi_{1}^{0}, \phi_{2}^{0}\right]},
\end{aligned}
$$

and the time step $\tau>0$, we consider the following first-order S-SAV scheme.

Scheme 3.1. Let $i=1,2$. Having computed $\left(\phi_{i}, r\right)^{k}$, we update $\left(\phi_{i}, r\right)^{k+1}$ by solving

$$
\begin{aligned}
& \frac{\phi_{i}^{k+1}-\phi_{i}^{k}}{\tau}=D_{i} \Delta \mu_{i}^{k+1}, \\
& \mu_{i}^{k+1}=-s_{i} \Delta\left(\phi_{i}^{k+1}-\phi_{i}^{k}\right)+\frac{r^{k+1}}{\sqrt{F\left[\phi_{1}^{k}, \phi_{2}^{k}\right]}} U_{i}\left[\phi_{1}^{k}, \phi_{2}^{k}\right], \\
& \frac{r^{k+1}-r^{k}}{\tau}=\frac{1}{2 \sqrt{F\left[\phi_{1}^{k}, \phi_{2}^{k}\right]}} \int_{\Omega} \sum_{j=1}^{2} U_{j}\left[\phi_{1}^{k}, \phi_{2}^{k}\right] \frac{\phi_{j}^{k+1}-\phi_{j}^{k}}{\tau} d \mathbf{x} .
\end{aligned}
$$

The energy dissipation law can be obtained similar to Theorem 3.1.

Theorem 3.2. For the modified energy

$$
\tilde{F}[r]=r^{2},
$$

the scheme (3.2a)-(3.2c) is unconditionally energy stable - i.e.

$$
\frac{1}{\tau}\left(\tilde{F}\left[r^{k+1}\right]-\tilde{F}\left[r^{k}\right]\right)=\frac{1}{\tau}\left(\left(r^{k+1}\right)^{2}-\left(r^{k}\right)^{2}\right) \leq 0 .
$$


Proof. Considering the inner product of the functions (3.2a) and multiplying (3.2c) by $2 r^{k+1}$, we have

$$
\begin{aligned}
\sum_{i=1}^{2}\left(\frac{\phi_{i}^{k+1}-\phi_{i}^{k}}{\tau}, \mu_{i}^{k+1}\right)= & \sum_{i=1}^{2} D_{i}\left(\Delta \mu_{i}^{k+1}, \mu_{i}^{k+1}\right), \\
\sum_{i=1}^{2}\left(\frac{\phi_{i}^{k+1}-\phi_{i}^{k}}{\tau}, \mu_{i}^{k+1}\right)= & \sum_{i=1}^{2}-s_{i}\left(\frac{\phi_{i}^{k+1}-\phi_{i}^{k}}{\tau}, \Delta\left(\phi_{i}^{k+1}-\phi_{i}^{k}\right)\right) \\
& +\sum_{i=1}^{2}\left(\frac{\phi_{i}^{k+1}-\phi_{i}^{k}}{\tau}, \frac{r^{k+1}}{\sqrt{F\left[\phi_{1}^{k}, \phi_{2}^{k}\right]}} U_{i}\left[\phi_{1}^{k}, \phi_{2}^{k}\right]\right), \\
\frac{\left(2\left(r^{k+1}\right)^{2}-2 r^{k+1} \cdot r^{k}\right)}{\tau}= & \sum_{i=1}^{2}\left(\frac{r^{k+1}}{\sqrt{F\left[\phi_{1}^{k}, \phi_{2}^{k}\right]}} U_{i}\left[\phi_{1}^{k}, \phi_{2}^{k}\right], \frac{\phi_{i}^{k+1}-\phi_{i}^{k}}{\tau}\right) .
\end{aligned}
$$

The terms

$$
\sum_{i=1}^{2}\left(\frac{\phi_{i}^{k+1}-\phi_{i}^{k}}{\tau}, \mu^{k+1}\right), \quad \sum_{i=1}^{2}\left(\frac{r^{k+1}}{\sqrt{F\left[\phi_{1}^{k}, \phi_{2}^{k}\right]}} U_{i}\left[\phi_{1}^{k}, \phi_{2}^{k}\right], \frac{\phi_{i}^{k+1}-\phi_{i}^{k}}{\tau}\right)
$$

are eliminated by summing them, so that

$$
\begin{aligned}
0 & \geq \sum_{i=1}^{2} D_{i}\left(\Delta \mu_{i}^{k+1}, \mu_{i}^{k+1}\right) \\
& =\sum_{i=1}^{2}-\frac{s_{i}}{\tau}\left(\phi_{i}^{k+1}-\phi_{i}^{k}, \Delta\left(\phi_{i}^{k+1}-\phi_{i}^{k}\right)\right)+\frac{1}{\tau}\left(r^{k+1}-r^{k}\right)^{2}+\frac{1}{\tau}\left(\left(r^{k+1}\right)^{2}-\left(r^{k}\right)^{2}\right) .
\end{aligned}
$$

Since the terms $\sum_{i=1}^{2}\left(-s_{i} / \tau\right)\left(\phi_{i}^{k+1}-\phi_{i}^{k}, \Delta\left(\phi_{i}^{k+1}-\phi_{i}^{k}\right)\right)$ and $\left(r^{k+1}-r^{k}\right)^{2} / \tau$ are nonnegative, the energy dissipation law follows.

\subsection{Solving the scheme}

In order to find solutions of the Eqs. (3.2a)-(3.2c), we write them in a different form. Substituting (3.2b) and (3.2c) into (3.2a) gives

$$
\mathscr{A}_{i} \phi_{i}^{k+1}=\mathscr{A}_{i} \phi_{i}^{k}+D_{i}\left(r^{k}-\frac{b^{k}}{2}\right) \Delta \xi_{i}^{k}+\frac{D_{i} b^{k+1}}{2} \Delta \xi_{i}^{k}
$$

where

$$
\begin{array}{ll}
\mathscr{A}_{i}=\frac{1}{\tau}+D_{i} s_{i} \Delta^{2}, & \xi_{i}^{k}=\frac{U_{i}\left[\phi_{1}^{k}, \phi_{2}^{k}\right]}{\sqrt{F\left[\phi_{1}^{k}, \phi_{2}^{k}\right]}} \\
b^{k}=\sum_{j=1}^{2}\left(\xi_{j}^{k}, \phi_{j}^{k}\right), & b^{k+1}=\sum_{j=1}^{2}\left(\xi_{j}^{k}, \phi_{j}^{k+1}\right) .
\end{array}
$$


The Eq. (3.3) yields

$$
\phi_{i}^{k+1}=\phi_{i}^{k}+D_{i}\left(r^{k}-\frac{b^{k}}{2}\right) \eta_{i}^{k}+\frac{D_{i} b^{k+1}}{2} \eta_{i}^{k}
$$

where

$$
\eta_{i}^{k}=\mathscr{A}_{i}^{-1}\left(\Delta \xi_{i}^{k}\right) .
$$

We can update $\phi_{i}^{k+1}$ by (3.5), but $b^{k+1}$ has to be updated first. Considering the inner products of (3.5) and $\xi_{i}^{k}, i=1,2$ and summing them, we obtain

$$
b^{k+1}=b^{k}+\left(r^{k}-\frac{b^{k}}{2}\right) c^{k}+\frac{c^{k}}{2} b^{k+1},
$$

where

$$
c^{k}=\sum_{i=1}^{2} D_{i}\left(\xi_{i}^{k}, \eta_{i}^{k}\right) .
$$

Subsequently, $b^{k+1}$ is updated as

$$
b^{k+1}=\frac{b^{k}+\left(r^{k}-b^{k} / 2\right) c^{k}}{1-c^{k} / 2} .
$$

Substituting $b^{k+1}$ into (3.5), we obtain $\phi_{i}^{k+1}$.

To summarise, the term $\phi_{i}^{k+1}$ is updated as follow.

\section{Algorithm 3.1}

1. Solve two linear equations to obtain $\eta_{i}^{k}$ from (3.6), $i=1,2$.

2. Compute $b^{k}$ from (3.4) and $c^{k}$ from (3.7).

3. Compute $b^{k+1}$ from (3.8).

4. Compute $\phi_{i}^{k+1}$ from (3.5), $i=1,2$.

Thus we only have to solve two linear equations with constant coefficients instead of two coupled fourth-order nonlinear parabolic partial differential equations.

\section{Simulations}

In this section, we present the results of numerical experiments to show the accuracy of the scheme, the mass conservation of the volume fractions, the decrease in the energy, and the influence of different parameters. The parameters are set according to Table 2. All parameters, except the statistical segment lengths $a_{i}$ in Subsection 4.5, are fixed through the numerical experiment. 
Table 2: The value of the parameters in the simulation.

\begin{tabular}{||l|c|c||}
\hline Parameter & Notation & Value \\
\hline \multirow{2}{*}{ The stabilising coefficients } & $s_{1}$ & 5 \\
\cline { 2 - 3 } & $s_{2}$ & 5 \\
\hline \multirow{2}{*}{ The mobility coefficients } & $D_{1}$ & 1 \\
\cline { 2 - 3 } & $D_{2}$ & 1 \\
\hline The volume of one MMS & $M$ & 0.16 \\
\hline The polymerization degree & $N$ & 4.34 \\
\hline \multirow{3}{*}{ The interaction parameters } & $\chi_{12}$ & 2 \\
\cline { 2 - 3 } & $\chi_{13}$ & 10 \\
\cline { 2 - 3 } & $\chi_{23}$ & 1.6 \\
\hline \multirow{3}{*}{ The statistical segment lengths } & $a_{1}$ & 1 \\
\cline { 2 - 3 } & $a_{2}$ & 1 \\
\cline { 2 - 3 } & $a_{3}$ & 1 \\
\hline
\end{tabular}

Remark 4.1. The second-order BDF S-SAV scheme for the MMC-TDGL equations is constructed, and the same numerical experiments have been carried out. Since the results for two schemes are similar, we present the results for the first-order S-SAV scheme only.

\subsection{Accuracy test}

We first test the convergence rates of the first-order S-SAV scheme, and we present the results of two tests with different initial conditions.

In the first test we consider the domain $\Omega=[0,64] \times[0,64]$ and the initial conditions

$$
\begin{aligned}
& \phi_{1}(x, y, 0)=0.1+0.01 \cos \left(\frac{6 \pi x}{64}\right) \cos \left(\frac{6 \pi y}{64}\right), \\
& \phi_{2}(x, y, 0)=0.5+0.01 \cos \left(\frac{6 \pi x}{64}\right) \cos \left(\frac{6 \pi y}{64}\right) .
\end{aligned}
$$

Here, the $256 \times 256$ uniform grid is used, so that the space step is 0.25 . Setting $\delta t=1.25 \times$ $10^{-5}$, we choose the numerical solution computed with the time step $\delta t / 12.5=1.0 \times 10^{-6}$ at $T=2.6$ as the reference solution. Numerical solutions are calculated with time steps $\tau=\delta t, \tau=2 \delta t, \tau=4 \delta t, \tau=8 \delta t, \tau=16 \delta t, \tau=32 \delta t$ at $T=1.6$. Table 3 shows the $L^{\infty}$. errors, $L^{2}$-errors and the convergence order of $\phi_{1}, \phi_{2}$ and $r$ with respect to the reference solution for different time steps. In Table $3, e_{i}(\tau)$ represents the errors for the time step $\tau$, i.e.

$$
\begin{aligned}
e_{i}(\tau) & =\phi_{i e}-\phi_{i \tau}, \quad i=1,2, \\
e_{r}(\tau) & =r_{e}-r_{\tau},
\end{aligned}
$$

where $\phi_{1 e}, \phi_{2 e}, r_{e}$ are the reference solutions, $\phi_{1 \tau}, \phi_{2 \tau}, r_{\tau}$ the numerical solutions for the time step $\tau$, and $\|\cdot\|_{\infty}$ and $\|\cdot\|_{2}$ are the $L^{\infty}$ - and $L^{2}$-norms, respectively. 
Table 3: Errors and convergence rates for $\phi_{1}, \phi_{2}$ and $r$ with the initial conditions (4.1).

\begin{tabular}{||c|c|c|c|c|c|c|c||}
\hline \multicolumn{2}{|c|}{$\tau$} & $32 \delta t$ & $16 \delta t$ & $8 \delta t$ & $4 \delta t$ & $2 \delta t$ & $\delta t$ \\
\hline \multirow{4}{*}{$\phi_{1}$} & $\left\|e_{1}(\tau)\right\|_{\infty}$ & $1.5077 \mathrm{e}-7$ & $7.5075 \mathrm{e}-8$ & $3.7275 \mathrm{e}-8$ & $1.8280 \mathrm{e}-8$ & $8.8792 \mathrm{e}-9$ & $4.2269 \mathrm{e}-9$ \\
\cline { 2 - 8 } & Order & & 1.0060 & 1.0101 & 1.0279 & 1.0417 & 1.0708 \\
\cline { 2 - 8 } & $\left\|e_{1}(\tau)\right\|_{2}$ & $3.6982 \mathrm{e}-6$ & $1.8461 \mathrm{e}-6$ & $9.1999 \mathrm{e}-7$ & $4.5713 \mathrm{e}-7$ & $2.2628 \mathrm{e}-7$ & $1.1070 \mathrm{e}-7$ \\
\cline { 2 - 8 } & Order & & 1.0023 & 1.0048 & 1.0090 & 1.0145 & 1.0314 \\
\hline \multirow{4}{*}{$\phi_{2}$} & $\left\|e_{2}(\tau)\right\|_{\infty}$ & $1.0005 \mathrm{e}-6$ & $4.9805 \mathrm{e}-7$ & $2.4727 \mathrm{e}-7$ & $1.2116 \mathrm{e}-7$ & $5.8679 \mathrm{e}-8$ & $2.7773 \mathrm{e}-8$ \\
\cline { 2 - 8 } & Order & & 1.0064 & 1.0102 & 1.0292 & 1.0460 & 1.0792 \\
\cline { 2 - 8 } & $\left\|e_{2}(\tau)\right\|_{2}$ & $2.4457 \mathrm{e}-5$ & $1.2206 \mathrm{e}-5$ & $6.0813 \mathrm{e}-6$ & $3.0185 \mathrm{e}-6$ & $1.4908 \mathrm{e}-6$ & $7.2600 \mathrm{e}-07$ \\
\cline { 2 - 8 } & Order & & 1.0027 & 1.0051 & 1.0106 & 1.0177 & 1.0380 \\
\hline \multirow{3}{*}{$r$} & $\left\|e_{r}(\tau)\right\|_{\infty}$ & $1.5126 \mathrm{e}-5$ & $7.5351 \mathrm{e}-6$ & $3.7442 \mathrm{e}-6$ & $1.8499 \mathrm{e}-6$ & $9.0298 \mathrm{e}-7$ & $4.2965 \mathrm{e}-7$ \\
\cline { 2 - 8 } & Order & & 1.0090 & 1.0172 & 1.0172 & 1.0347 & 1.0715 \\
\hline
\end{tabular}

In the second test, we consider the domain $\Omega=[0,50] \times[0,50]$ and use the uniform $200 \times 200$ grid with the same space step 0.25 . The initial conditions

$$
\begin{aligned}
& \phi_{1}(x, y, 0)=0.1+\zeta_{1}(x, y), \\
& \phi_{2}(x, y, 0)=0.5+\zeta_{2}(x, y)
\end{aligned}
$$

with the uniformly distributed on $[0,0.01]$ random terms $\zeta_{i}(x, y), i=1,2$, are computed at $T=1$ with the time step $\tau=0.001$. The numerical solution at $T=1$ is the true initial condition we set in the first test. The purpose of this step is to avoid disturbances from the random terms. Setting $\delta t=1.25 \times 10^{-5}$, we choose the numerical solution computed with the time step $\delta t / 12.5=1.0 \times 10^{-6}$ at $T=2.6$ as the reference solution. Then, the numerical solutions are computed with the time step $\tau=\delta t, \tau=2 \delta t, \tau=4 \delta t, \tau=$ $8 \delta t, \tau=16 \delta t, \tau=32 \delta t$ at $T=2.6$. The notations in Table 4 are the same as in Table 3 . Tables 3 and 4 show that the first-order S-SAV scheme matches the first-order accuracy in time.

Table 4: Errors and convergence rates for $\phi_{1}, \phi_{2}$ and $r$ with the initial conditions (4.2).

\begin{tabular}{||c|c|c|c|c|c|c|c||}
\hline \multicolumn{2}{||c|}{$\tau$} & $32 \delta t$ & $16 \delta t$ & $8 \delta t$ & $4 \delta t$ & $2 \delta t$ & $\delta t$ \\
\hline \multirow{4}{*}{$\phi_{1}$} & $\left\|e_{1}(\tau)\right\|_{\infty}$ & $1.3682 \mathrm{e}-4$ & $6.9398 \mathrm{e}-5$ & $3.4820 \mathrm{e}-5$ & $1.7309 \mathrm{e}-5$ & $8.4960 \mathrm{e}-6$ & $4.0754 \mathrm{e}-6$ \\
\cline { 2 - 8 } & Order & & 0.9545 & 0.9793 & 0.9950 & 1.0266 & 1.0598 \\
\cline { 2 - 8 } & $\left\|e_{1}(\tau)\right\|_{2}$ & $1.6131 \mathrm{e}-3$ & $8.1574 \mathrm{e}-4$ & $4.0867 \mathrm{e}-4$ & $2.0299 \mathrm{e}-4$ & $9.9587 \mathrm{e}-5$ & $4.7765 \mathrm{e}-5$ \\
\cline { 2 - 8 } & Order & & 0.9837 & 0.9972 & 1.0096 & 1.0272 & 1.0601 \\
\hline \multirow{4}{*}{$\phi_{2}$} & $\left\|e_{2}(\tau)\right\|_{\infty}$ & $9.4696 \mathrm{e}-4$ & $4.8055 \mathrm{e}-4$ & $2.4118 \mathrm{e}-4$ & $1.1990 \mathrm{e}-4$ & $5.8857 \mathrm{e}-5$ & $2.8234 \mathrm{e}-5$ \\
\cline { 2 - 8 } & Order & & 0.9786 & 0.9946 & 1.0083 & 1.0265 & 1.0598 \\
\cline { 2 - 8 } & $\left\|e_{2}(\tau)\right\|_{2}$ & $1.0762 \mathrm{e}-2$ & $5.4435 \mathrm{e}-3$ & $2.7273 \mathrm{e}-3$ & $1.3547 \mathrm{e}-3$ & $6.6472 \mathrm{e}-4$ & $3.1880 \mathrm{e}-4$ \\
\cline { 2 - 8 } & Order & & 0.9951 & 1.0030 & 1.0125 & 1.0287 & 1.0609 \\
\hline \multirow{3}{*}{$r$} & $\left\|e_{r}(\tau)\right\|_{\infty}$ & $1.9194 \mathrm{e}-5$ & $9.7429 \mathrm{e}-6$ & $4.8901 \mathrm{e}-6$ & $2.4310 \mathrm{e}-6$ & $1.1931 \mathrm{e}-6$ & $5.7199 \mathrm{e}-7$ \\
\cline { 2 - 8 } & Order & & 0.9783 & 0.9945 & 1.0083 & 1.0268 & 1.0607 \\
\hline
\end{tabular}




\subsection{Influence of the initial concentration}

Here, we show the phase transition processes for different initial concentrations. In later simulations, we considered the domain $\Omega=[0,50] \times[0,50]$ with the uniform $200 \times 200$ grid. We now choose time step $\tau=0.001$ and the initial conditions

$$
\begin{aligned}
& \phi_{1}(x, y, 0)=\phi_{10}+\zeta_{1}(x, y), \\
& \phi_{2}(x, y, 0)=\phi_{20}+\zeta_{2}(x, y),
\end{aligned}
$$

where $\phi_{10}, \phi_{20}$ are constants and $\zeta_{i}(x, y), i=1,2$ are uniformly distributed random terms on $[0,0.01]$.

In the first experiment, we fix $\phi_{10}=0.1$. Figs. $2-5$ show the phase transition process of $\phi_{20}=0.2,0.3,0.4,0.5$. In each figure, the four columns of the graphs are taken at $t=5,50,100,200$, and the three rows of the graphs show the evolution of the volume fraction variable $\phi_{1}, \phi_{2}, \phi_{1}+\phi_{2}$. The light blue domain in the first row, corresponding to the larger values of $\phi_{1}$, represents the higher concentration of MMSs. The yellow domain in the second row, corresponding to the larger values of $\phi_{2}$, represents the concentrated polymer segments. The red domain in the third row, corresponding to the larger values of $\phi_{1}+\phi_{2}$, represents the higher concentration of the composition of MMSs and polymer chains.

For low initial concentrations of polymer segments, the polymer chains cannot grow long enough to graft onto other MMSs or polymer chains - cf. Fig. 2. Therefore, MMSs and polymer chains form isolated balls. If the initial concentration of the polymer segments is increased to $\phi_{20}=0.3$, some balls can be joined by polymer chains and form a bar - cf.
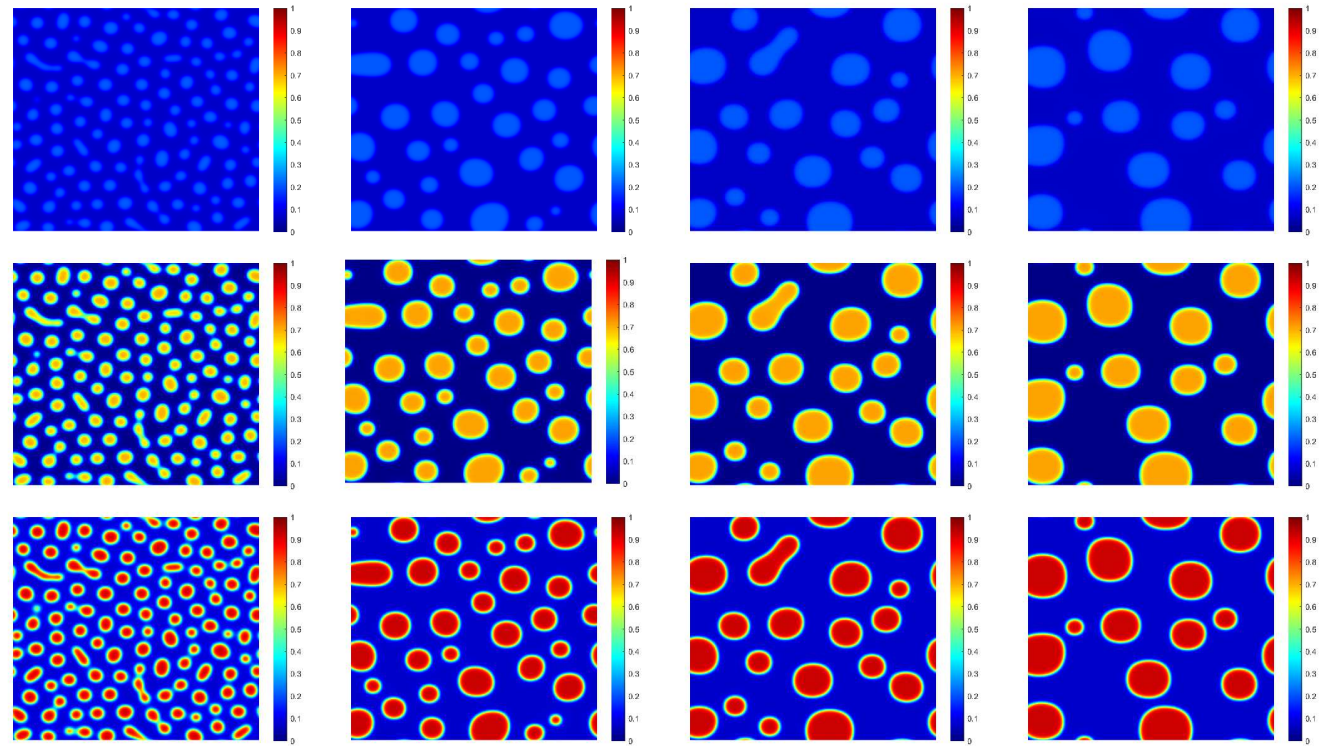

Figure 2: Evolution in the case $\phi_{10}=0.1, \phi_{20}=0.2$. The rows show the evolution of the volume fraction variables $\phi_{1}, \phi_{2}, \phi_{1}+\phi_{2}$. The columns are taken at $t=5,50,100,200$. 

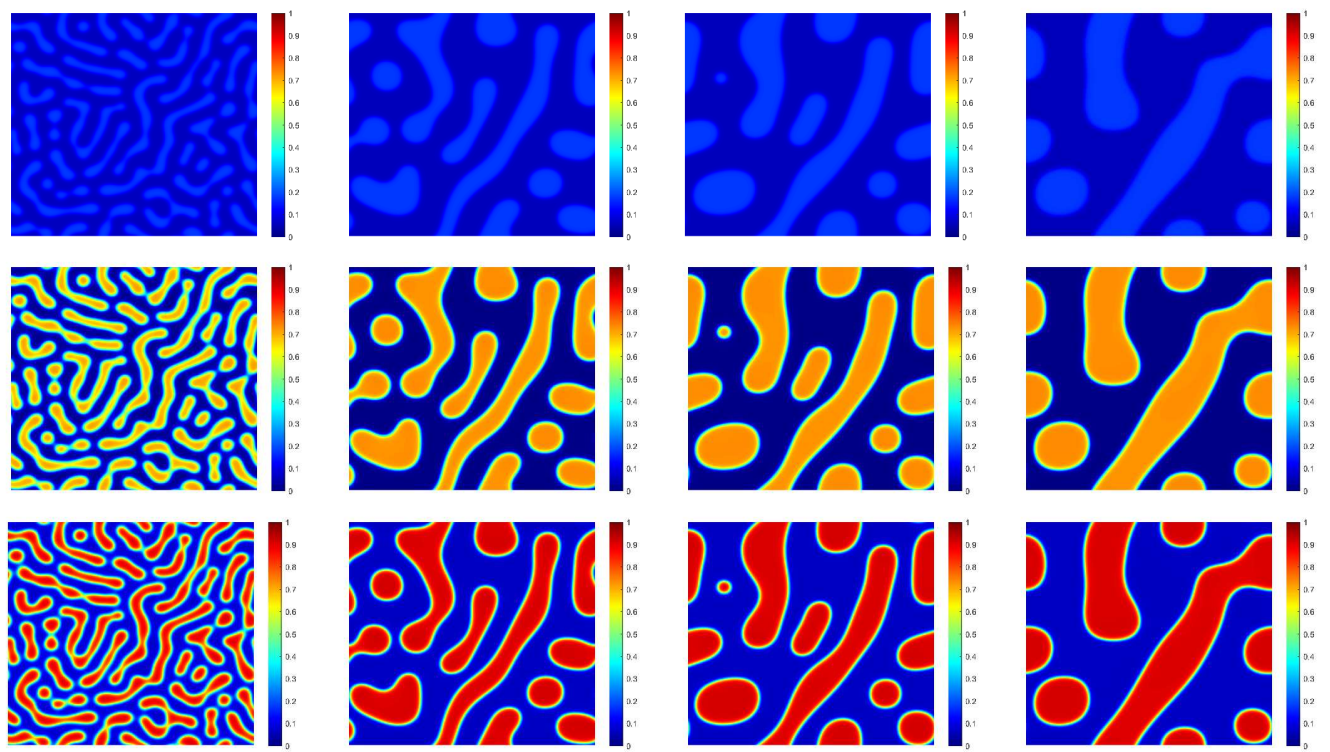

Figure 3: Evolution in the case $\phi_{10}=0.1, \phi_{20}=0.3$. The rows show the evolution of the volume fraction variables $\phi_{1}, \phi_{2}, \phi_{1}+\phi_{2}$. The columns are taken at $t=5,50,100,200$.
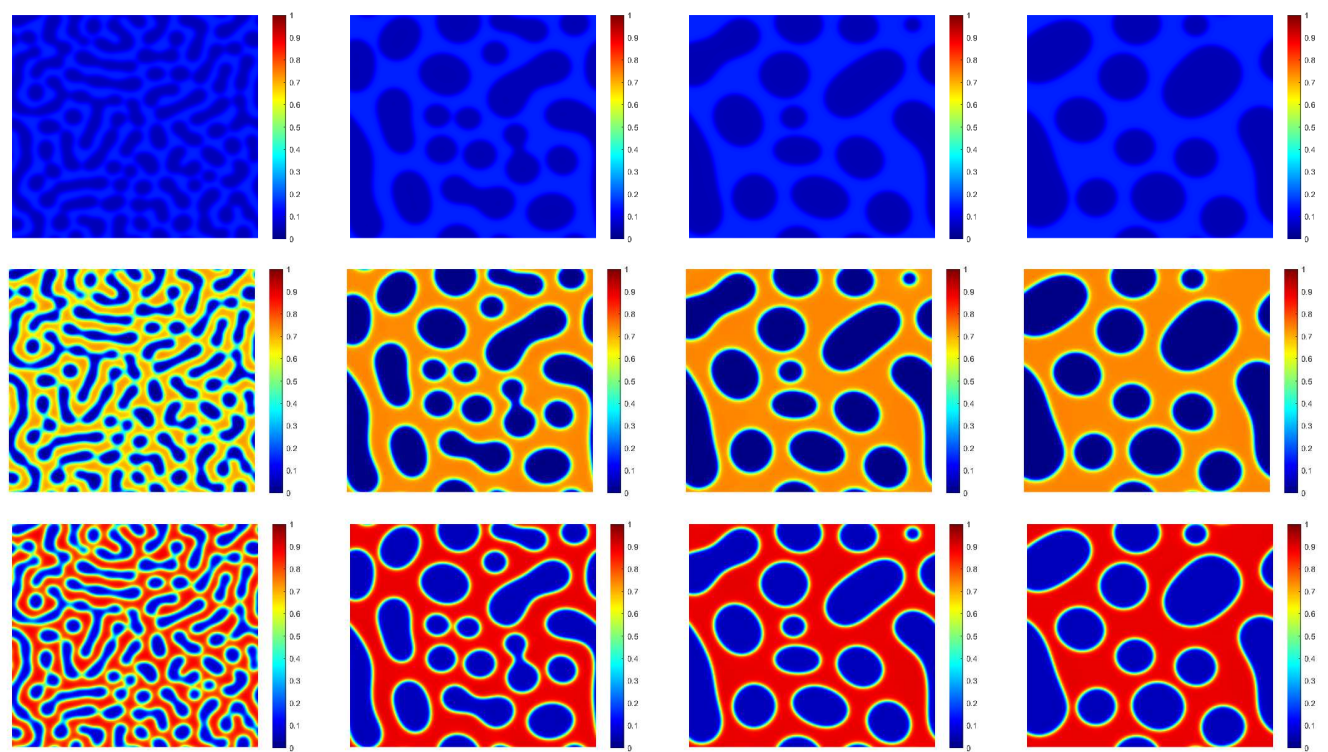

Figure 4: Evolution in the case $\phi_{10}=0.1, \phi_{20}=0.4$. The rows show the evolution of the volume fraction variables $\phi_{1}, \phi_{2}, \phi_{1}+\phi_{2}$. The columns are taken at $t=5,50,100,200$.

Fig. 3. However, other balls are still separated due to the lack of polymer segments. If the initial concentration of polymer segments reaches $\phi_{20}=0.4$ and 0.5 , every MMS can be joined by polymer chains since there are enough segments for the polymer chains to grow 

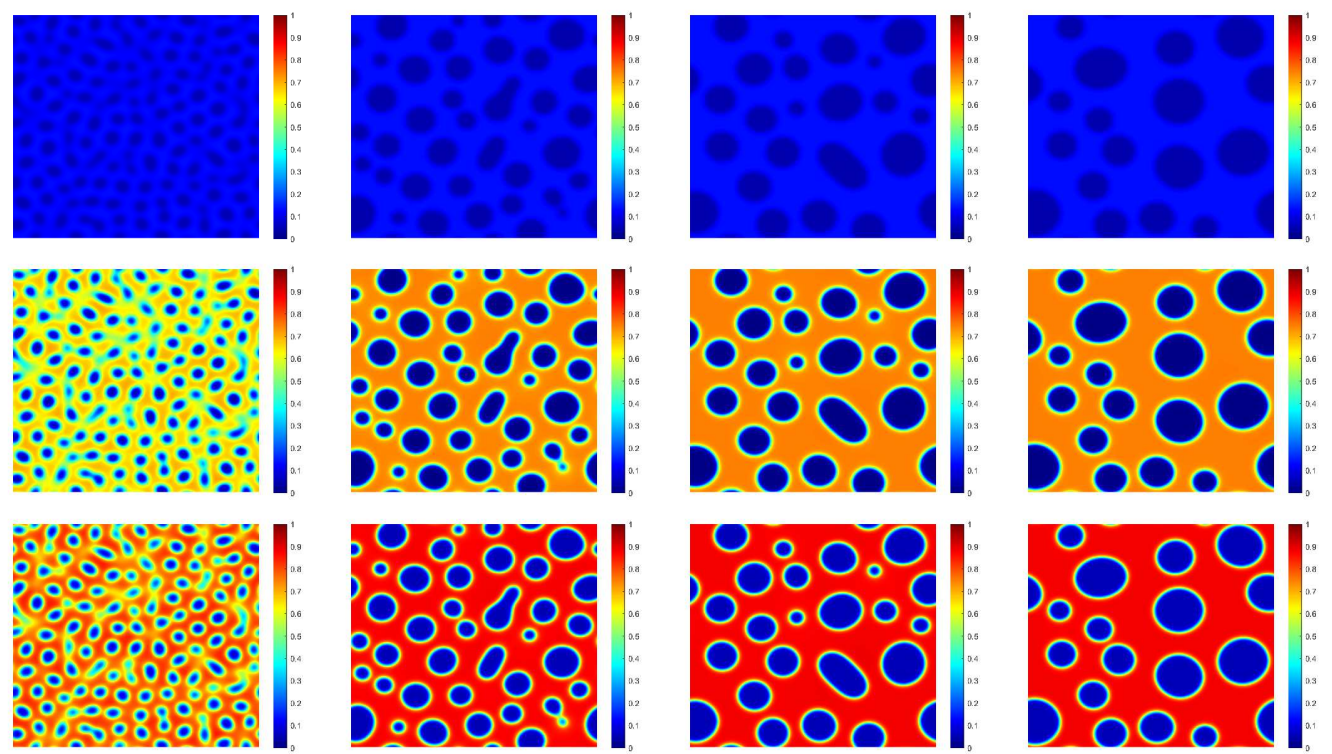

Figure 5: Evolution in the case $\phi_{10}=0.1, \phi_{20}=0.5$. The rows show the evolution of the volume fraction variables $\phi_{1}, \phi_{2}, \phi_{1}+\phi_{2}$. The columns are taken at $t=5,50,100,200$.
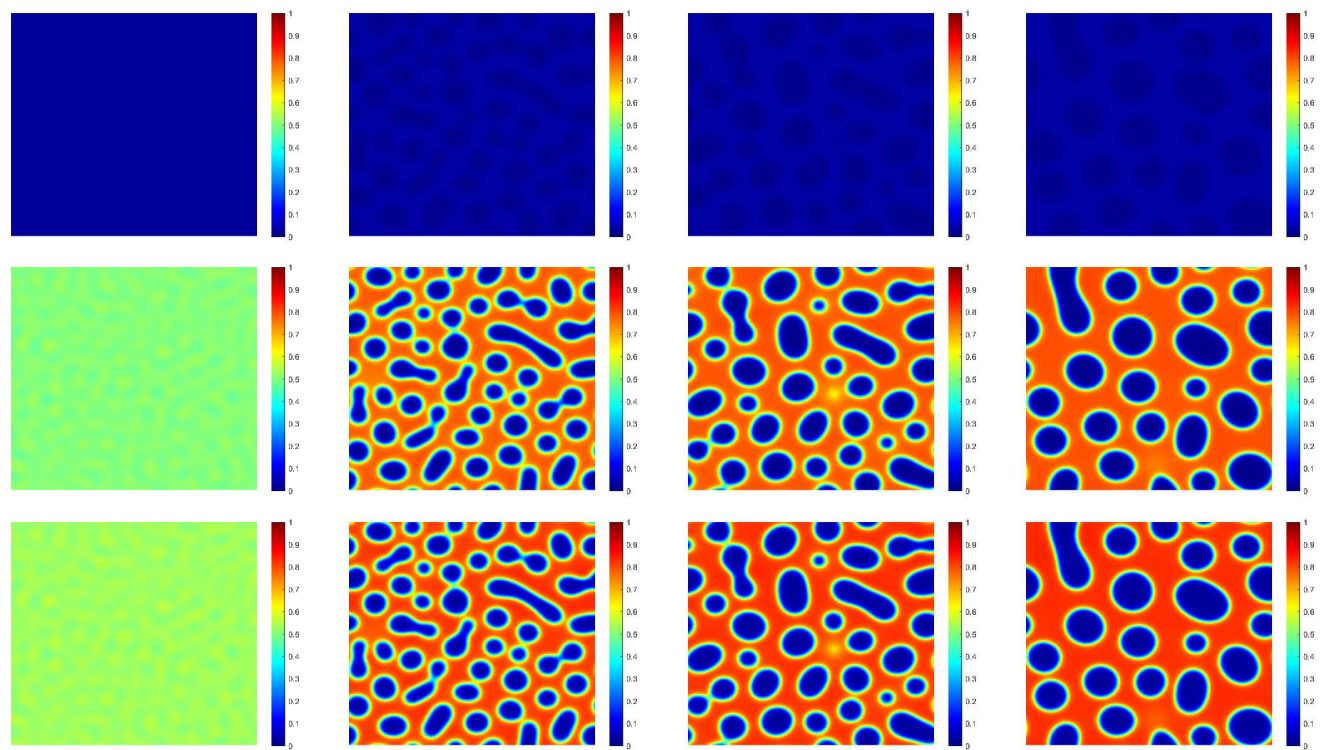

Figure 6: Evolution in the case $\phi_{10}=0.02, \phi_{20}=0.5$. The rows show the evolution of the volume fraction variables $\phi_{1}, \phi_{2}, \phi_{1}+\phi_{2}$. The columns are taken at $t=5,50,100,200$.

- cf. Figs. 4 and 5. Therefore, in these two cases, a reticular structure can be obtained. When the initial concentration is higher, the red area in the third row becomes larger i.e. the structure is tighter. This is consistent with the results in [29]. 

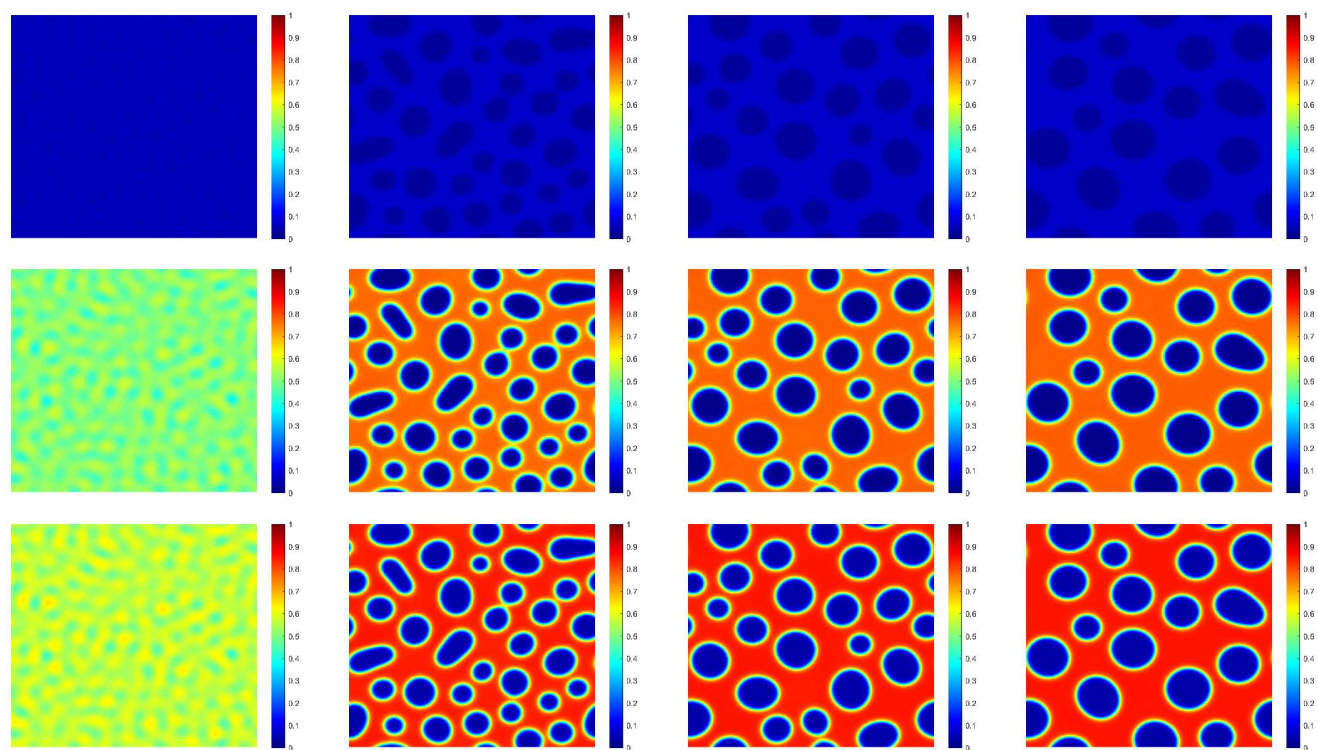

Figure 7: Evolution in the case $\phi_{10}=0.05, \phi_{20}=0.5$. The rows show the evolution of the volume fraction variables $\phi_{1}, \phi_{2}, \phi_{1}+\phi_{2}$. The columns are taken at $t=5,50,100,200$.
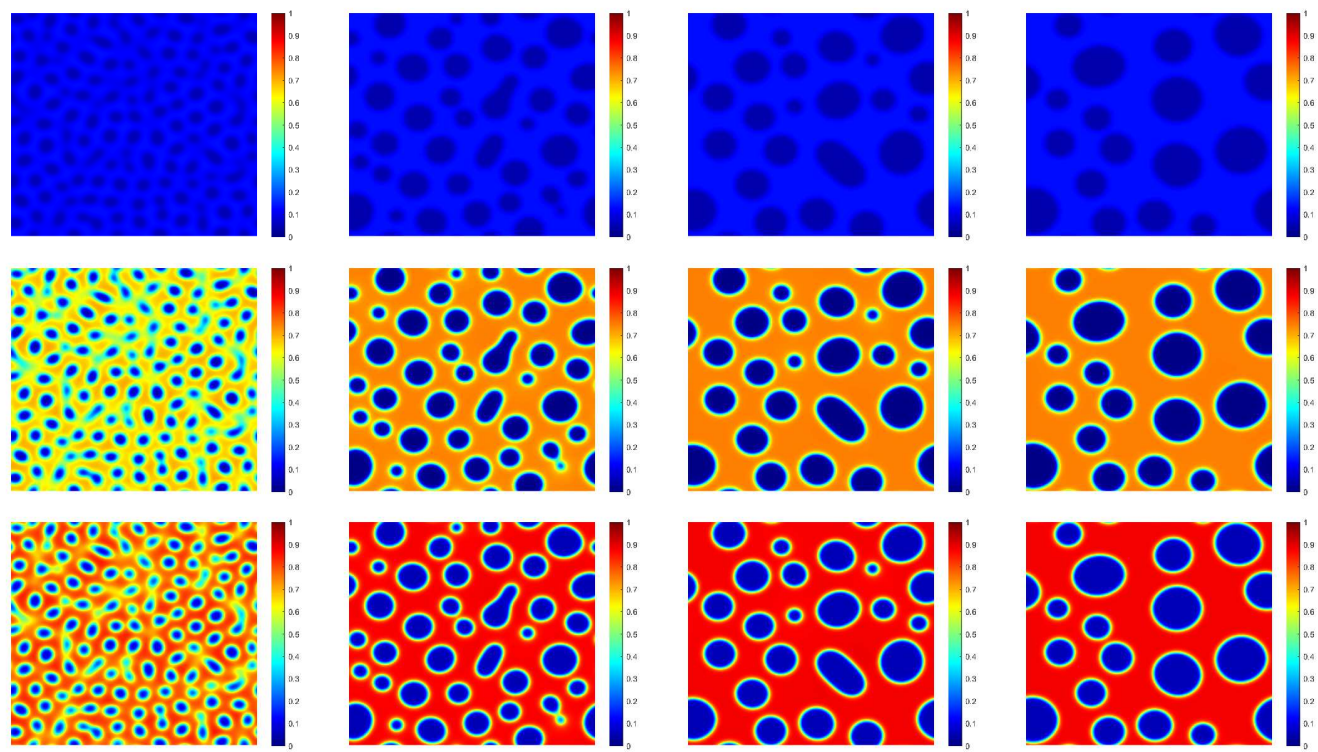

Figure 8: Evolution in the case $\phi_{10}=0.1, \phi_{20}=0.5$. The rows show the evolution of the volume fraction variables $\phi_{1}, \phi_{2}, \phi_{1}+\phi_{2}$. The columns are taken at $t=5,50,100,200$.

In the second experiment, we fix $\phi_{20}=0.5$. Fig. 6-9 show the phase transition of $\phi_{10}=0.02,0.05,0.1,0.15$. Note that if the initial concentration of the MMSs increases, the light blue domain in the first row turns lighter. This indicates that the concentration of MMSs increases. The orange domain in the second row turns yellow, which indicates 

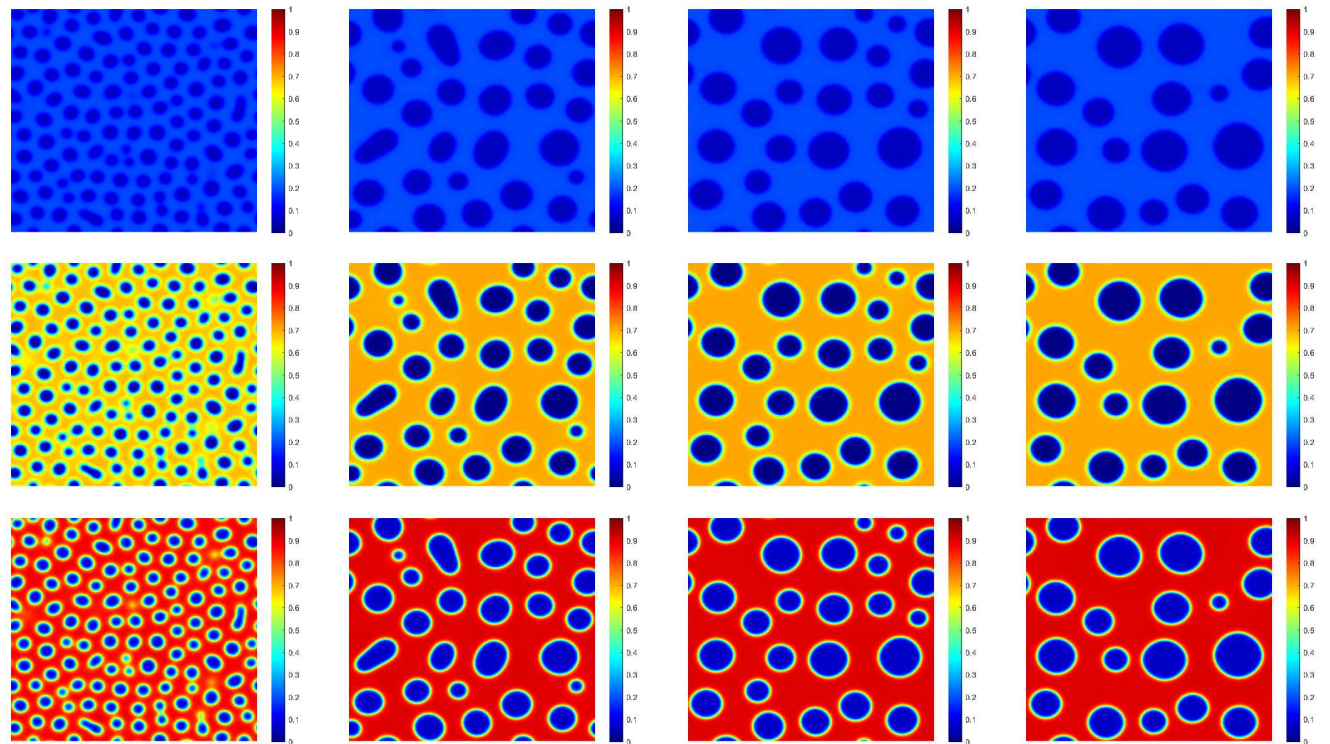

Figure 9: Evolution in the case $\phi_{10}=0.15, \phi_{20}=0.5$. The rows show the evolution of the volume fraction variables $\phi_{1}, \phi_{2}, \phi_{1}+\phi_{2}$. The columns are taken at $t=5,50,100,200$.

the concentration of polymer chains decreases. The red domain in the third row turns darker. This indicates that the concentration of the composition of MMSs and polymer chains increases. A reticular structure can be formed in all four cases - i.e. if the initial concentration of the polymer segments is sufficiently high, the initial concentration of the MMSs does not affect the formation of the structure.

\subsection{The evolution of total mass and modified energy}

In order to verify numerically the mass conservation of the MMC-TDGL equations in Theorem 2.2, we transform the integral $\int_{\Omega} \phi_{i} d \mathbf{x}$ into the discrete form $\sum_{m, n=1}^{200} \phi_{i, m, n} h^{2}$ and then plot the graph of the sums over time $t$. Fig. 10 a) shows the total mass of $\phi_{2}$ for initial concentrations $\phi_{20}=0.2,0.3,0.4,0.5$ in the first experiment in Subsection 4.2, and Fig. $10 \mathrm{~b}$ ) shows the total mass of $\phi_{1}$ for initial concentrations $\phi_{10}=0.02,0.05,0.1,0.15$ in the second experiment. The points lie on horizontal lines. This implies that the numerical solutions are mass conservative - i.e. the first-order S-SAV scheme maintains mass conservation.

The modified energy $\tilde{F}$ with time $t$ for different cases is plotted to verify the modified energy stability. Fig. 11 a) shows the time evolution of the modified energy for the initial concentrations $\phi_{20}=0.2,0.3,0.4,0.5$ in the first experiment, and Fig. $11 \mathrm{~b}$ ) shows the time evolution of the modified energy for the initial concentrations $\phi_{10}=0.02,0.05,0.1,0.15$ in the second experiment. All curves show a downward tendency - i.e. the modified energy decreases with time, as proved in Theorem 3.2. Fig. 12 demonstrates the original and modified energies for $\phi_{10}=0.05, \phi_{20}=0.5$. 


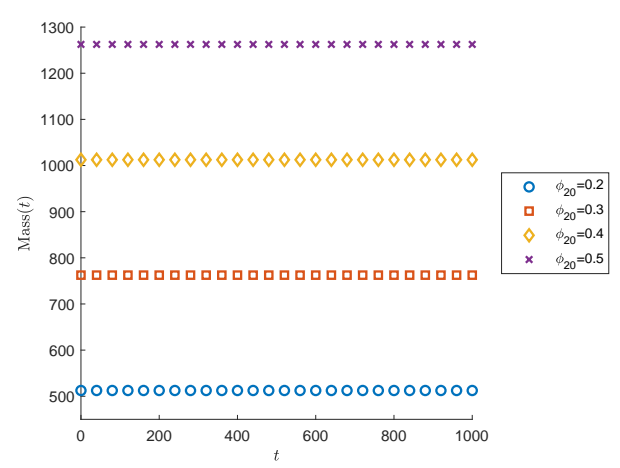

a)

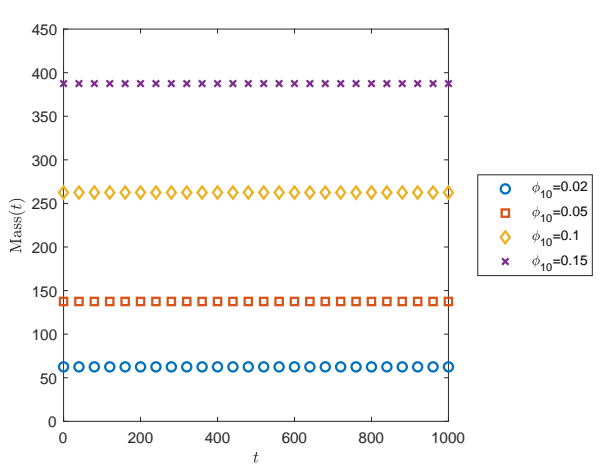

b)

Figure 10: Evolution of total mass. a) Total mass of $\phi_{2}$ for different initial concentrations $\phi_{20}=$ $0.2,0.3,0.4,0.5$. b) Total mass of $\phi_{1}$ for different initial concentrations $\phi_{10}=0.02,0.05,0.1,0.15$.

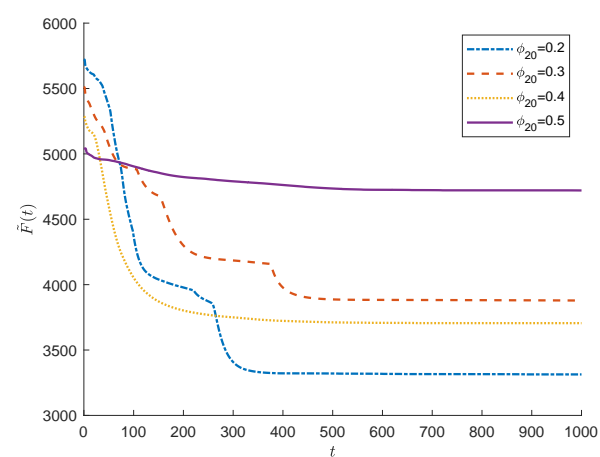

a)

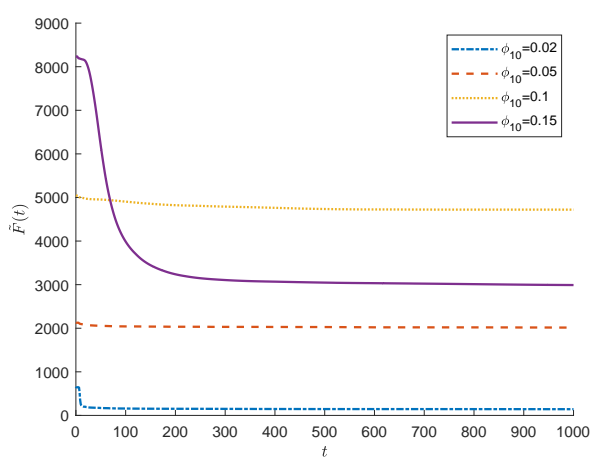

b)

Figure 11: Evolution of modified energy. a) Modified energy for initial concentrations $\phi_{20}=$ $0.2,0.3,0.4,0.5$. b) Modified energy for initial concentrations $\phi_{10}=0.02,0.05,0.1,0.15$.

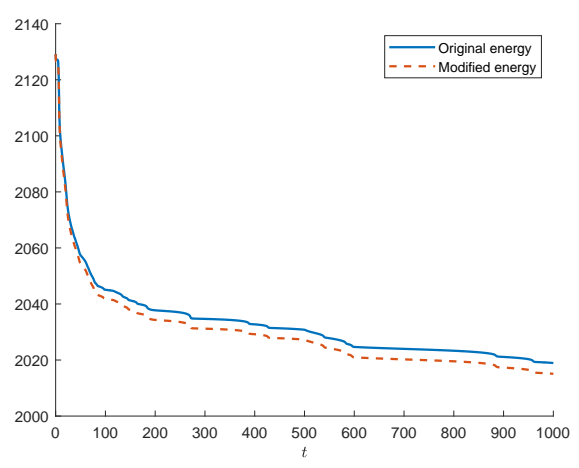

Figure 12: Original and modified energy for the case $\phi_{10}=0.05, \phi_{20}=0.5$.

\subsection{The steady state with time-developing}

Theorem 3.2 and Fig. 11 show that the modified energy $\tilde{F}$ decreases with time. The further evolution for $\phi_{10}=0.1, \phi_{20}=0.5$ is displayed in Fig. 13. We note that $\phi_{1}, \phi_{2}$ and 

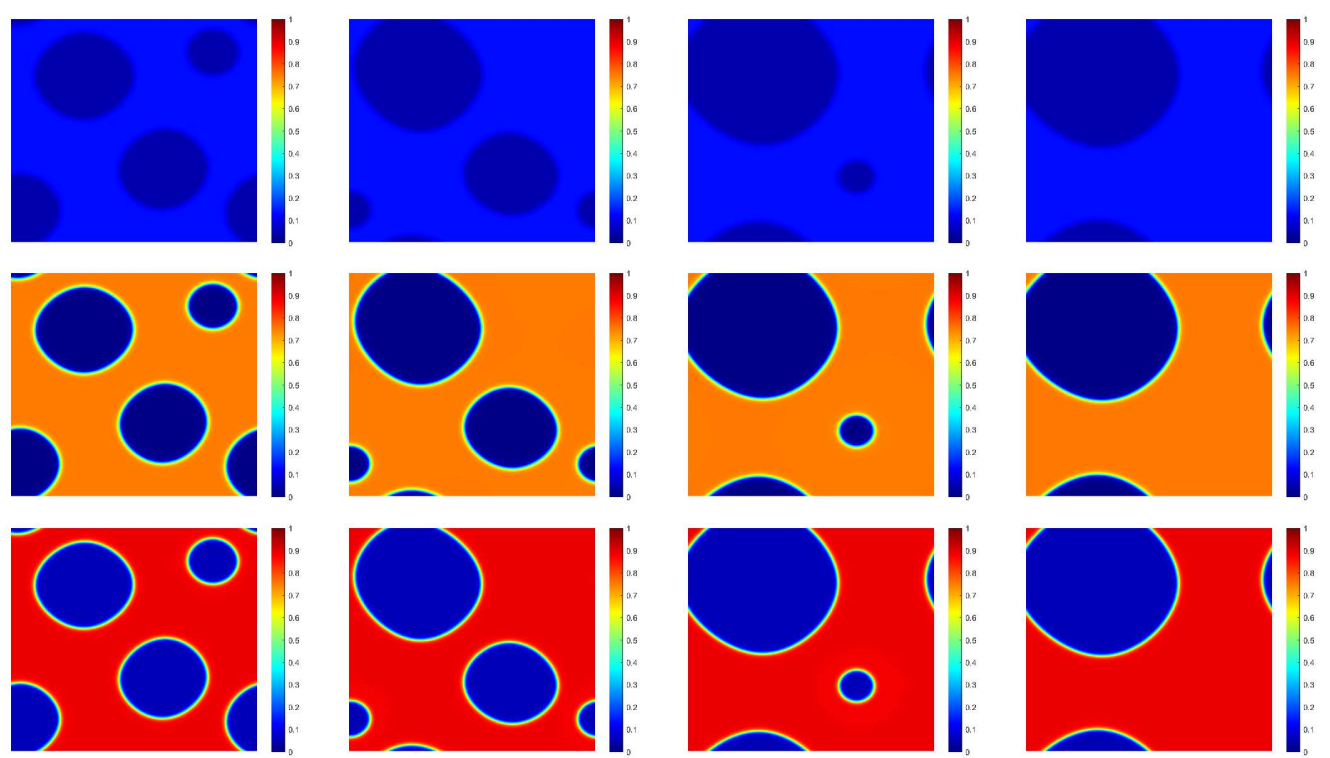

Figure 13: Evolution for $\phi_{10}=0.1, \phi_{20}=0.5$ and its steady state. The rows show the evolution of the volume fraction variables $\phi_{1}, \phi_{2}, \phi_{1}+\phi_{2}$. The columns are taken at $t=2000,5000,8000,8200$.

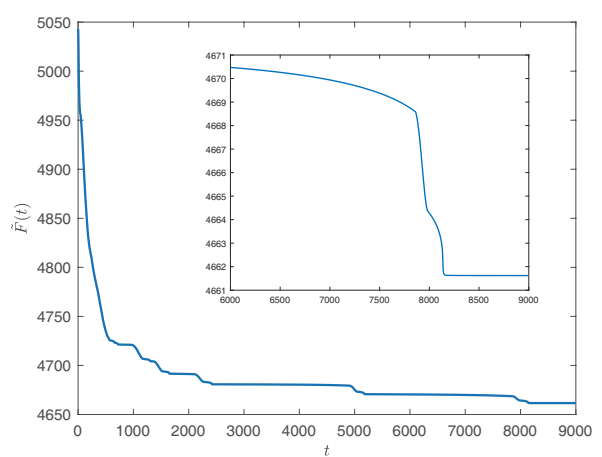

Figure 14: The modified energy in the steady state for the case $\phi_{10}=0.1, \phi_{20}=0.5$.

$\phi_{1}+\phi_{2}$ almost reach the steady state at $t=8200$. The modified energy in Fig. 14 stays conserved till it reaches the steady state. This is consistent with the results in [51].

\subsection{Influence of different statistical segment lengths $a_{i}$}

The statistical segment lengths $a_{i}, i=1,2,3$ in the interface gradient terms of de-Gennes $(1 / 36) \sum_{i=1}^{3}\left(a_{i}^{2} / \phi_{i}\right)\left|\nabla \phi_{i}\right|^{2}$ determine the thickness of the interface. The numerical experiments are aimed to verify this observation. We set the initial conditions

$$
\begin{aligned}
& \phi_{1}(x, y, 0)=0.1+\zeta_{1}(x, y), \\
& \phi_{2}(x, y, 0)=0.5+\zeta_{2}(x, y),
\end{aligned}
$$



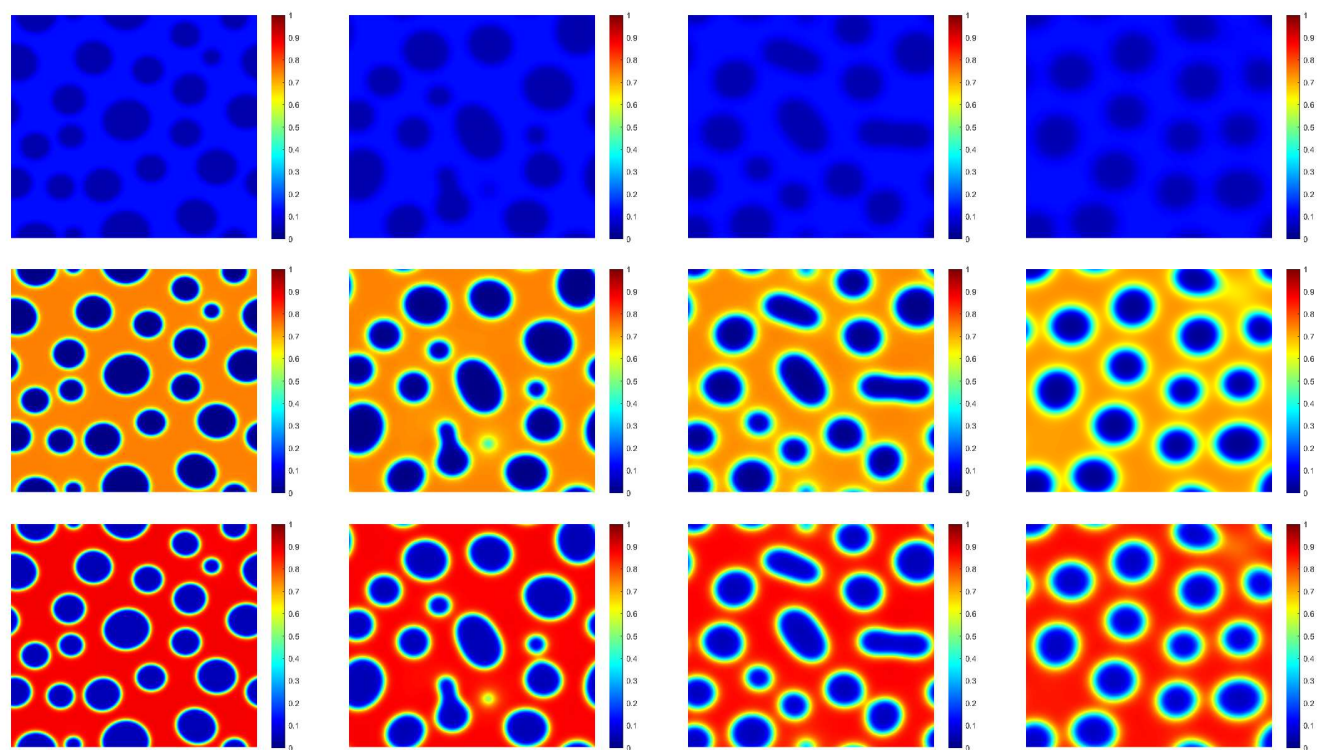

Figure 15: Numerical experiments with statistical segment lengths $a_{i}, i=1,2,3$. Columns present numerical results with $a_{i}=1,1.5,2,2.5$. Rows show evolution of volume fraction variables $\phi_{1}, \phi_{2}, \phi_{1}+\phi_{2}$.

where $\zeta_{i}(x, y), i=1,2$ are uniformly distributed random terms on $[0,0.01]$. The graphs in Fig. 15 are taken at $t=100$. The four columns present the numerical results with $a_{i}=1,1.5,2,2.5$. The rows of the graphs show the evolution of the volume fraction variable $\phi_{1}, \phi_{2}, \phi_{1}+\phi_{2}$. For clarity, the range of the axis is changed from $[0,1]$ to $[0,0.2]$ when the volume fraction $\phi_{1}$ is plotted. We note that the thickness of the interface increases as the statistical segment length $a_{i}$ increases.

\section{Conclusion}

Ternary MMC-TDGL equations and a first-order S-SAV scheme for these equations are constructed. Since an MMC hydrogel has a well-defined reticular structure, we use the Boltzmann entropy theorem, Flory-Huggins lattice theory and statistical thermodynamic method in order to develop a reticular free energy, which replaces the Flory-Huggins free energy. Combined with the TDGL method, the ternary MMC-TDGL equations are obtained based on the ternary Cahn-Hilliard equations. For the simulation of the MMC hydrogel phase transition, a first-order S-SAV scheme is constructed. It is linear and unconditionally energy stable. Using the S-SAV approach, the ternary MMC-TDGL equations are decoupled into two linear equations with constant coefficients, so the first-order S-SAV scheme is efficient. Numerical experiments illustrate the accuracy of the scheme, the mass conservation of the volume fractions, and the decrease in the modified energy. The results of simulations using different parameters are consistent with the physical phenomena. The convergence and error analysis of the first-order S-SAV scheme will be studied later on. 


\section{Acknowledgments}

G. Ji is partially supported by the National Natural Science Foundation of China (Grant Nos. 11571045, 11671052 and 11871105), the State Scholarship Fund of CSC (Grant No. 201406045016) and Fundamental Research Funds for the Central Universities (Grant No. 2015KJJCB23). H. Zhang is partially supported by the National Natural Science Foundation of China (Grant No. 11971002).

\section{References}

[1] A. Baskaran, Z. Hu, J.S. Lowengrub, C. Wang, S.M. Wise and P. Zhou, Energy stable and efficient finite-difference nonlinear multigrid schemes for the modified phase field crystal equation, J. Comput. Phys. 250, 270-292 (2013).

[2] F. Boyer and S. Minjeaud, Numerical schemes for a three component Cahn-Hilliard model, ESAIM Math. Model. Numer. Anal. 45(4), 697-738 (2011).

[3] C. Chen and X. Yang, Fast, provably unconditionally energy stable, and second-order accurate algorithms for the anisotropic Cahn-Hilliard model, Comput. Methods Appl. Mech. Engrg. 351, 35-59 (2019).

[4] R. Chen, G. Ji, X. Yang and H. Zhang, Decoupled energy stable schemes for phase-field vesicle membrane model, J. Comput. Phys. 302, 509-523 (2015).

[5] R. Chen, X. Yang and H. Zhang, Second order, linear, and unconditionally energy stable schemes for a hydrodynamic model of Smectic-A liquid crystals, SIAM J. Sci. Comput. 39(6), A2808A2833 (2017).

[6] W. Chen, S. Conde, C. Wang, X. Wang and S.M. Wise, A linear energy stable scheme for a thin film model without slope selection, J. Sci. Comput. 52(3), 546-562 (2012).

[7] W. Chen, C. Wang, X. Wang and S.M. Wise, A linear iteration algorithm for a second-order energy stable scheme for a thin film model without slope selection, J. Sci. Comput. 59(3), 574601 (2014).

[8] W. Chen, X. Wang, Y. Yang and Z. Zhang, A second order BDF numerical scheme with variable steps for the Cahn-Hilliard equation, SIAM J. Numer. Anal. 57(1), 495-525 (2019).

[9] K. Cheng, W. Feng, C. Wang and S.M. Wise, An energy stable fourth order finite difference scheme for the Cahn-Hilliard equation, J. Comput. Appl. Math. 362, 574-595 (2019).

[10] K. Cheng, C. Wang and S.M. Wise, An energy stable BDF2 Fourier pseudo-spectral numerical scheme for the square phase field crystal equation, Commun. Comput. Phys. 26(5), 1335-1364 (2019).

[11] Q. Cheng, C. Liu and J. Shen, A new Lagrange multiplier approach for gradient flows, Comput. Methods Appl. Mech. Engrg. 367, (2020).

[12] Q. Cheng, X. Yang and J. Shen, Efficient and accurate numerical schemes for a hydro-dynamically coupled phase field diblock copolymer model, J. Comput. Phys. 341, 44-60 (2017).

[13] P. Dayal and T. Kyu, Morphology development in polymer fibers undergoing solvent/non-solvent exchange, Macromol. Symp. 25, 170-178 (2007).

[14] P. Degennes, Dynamics of fluctuations and spinodal decomposition in polymer blends, J. Chem. Phys. 72(7), 4756-4763 (1980).

[15] C.M. Elliott and A.M. Stuart, The global dynamics of discrete semilinear parabolic equations, SIAM J. Numer. Anal. 30(6), 1622-1663 (1993).

[16] D.J. Eyre, Unconditionally gradient stable time marching the Cahn-Hilliard equation, Computational and Mathematical Models of Microstructural Evolution 529, $39-46$ (1998). 
[17] W. Feng, C. Wang, S.M. Wise and Z. Zhang, A second-order energy stable backward differentiation formula method for the epitaxial thin film equation with slope selection, Numer. Methods Partial Differential Equations. 34(6), 1975-2007 (2018).

[18] X. Feng, T. Tang and J. Yang, Long time numerical simulations for phase-field problems using padaptive spectral deferred correction methods, SIAM J. Sci. Comput. 37(1), A271-A294 (2015).

[19] S.C. Glotzer and W. Paul, Molecular and mesoscale simulation methods for polymer materials, Annu. Rev. Mater. Sci. 11(32), 401-436 (2002).

[20] R. Guo and Y. Xu, Efficient, accurate and energy stable discontinuous Galerkin methods for phase field models of two-phase incompressible flows, Commun. Comput. Phys. 26(4), 1224-1248 (2019).

[21] C. He, K. Jiao, X. Zhang, M. Xiang, Z. Li and H. Wang, Nanoparticles, microgels and bulk hydrogels with very high mechanical strength starting from micelles, Soft Matter. 7(6), 2943 (2011).

[22] M. He, H. Zhang, W. Chen and X. Dong, Polymer Physics, Fudan University Press (2012).

[23] J. Hu, K. Hiwatashi, T. Kurokawa, S. Liang, Z. Wu and J. Gong, Microgel-reinforced hydrogel films with high mechanical strength and their visible mesoscale fracture structure, Macromolecules 44(19), 7775-7781 (2011).

[24] Z. Hu, S.M. Wise, C. Wang and J. Lowengrub, Stable and efficient finite-difference nonlinearmultigrid schemes for the phase field crystal equation, J. Comput. Phys. 228(15), 5323-5339 (2009).

[25] T. Huang, H. Xu, K. Jiao, L. Zhu, H.R. Brown and H. Wang, A novel hydrogel with high mechanical strength: A macromolecular microsphere composite hydrogel, Adv. Mater. 19(12), 1622-1626 (2010).

[26] F. Jiang, T. Huang, C. He, H.R. Brown and H. Wang, Interactions affecting the mechanical properties of macromolecular microsphere composite hydrogels, J. Phys. Chem. B 117(43), 1367913687 (2013).

[27] F. Kang and Z. Zhang, A multiple scalar auxiliary variables approach to the energy stable scheme of the moving contact line problem, Numer. Math. Theory Methods Appl. 13(2), 539-568 (2020).

[28] D. Li and Z. Qiao, On second order semi-implicit Fourier spectral methods for 2D Cahn-Hilliard equations, J. Sci. Comput. 70(1), 301-341 (2017).

[29] X. Li, G. Ji and H. Zhang, Phase transitions of macromolecular microsphere composite hydrogels based on the stochastic Cahn-Hilliard equation, J. Comput. Phys. 283, 81-97 (2015).

[30] X. Li, Z. Qiao and H. Zhang, An unconditionally energy stable finite difference scheme for a stochastic Cahn-Hilliard equation, Sci. China Math. 59(9), 1815-1834 (2016).

[31] X. Li, J. Shen and H. Rui, Energy stability and convergence of SAV block-centered finite difference method for gradient flows, Math. Comp. 88(319), 2047-2068 (2019).

[32] L. Ma, R. Chen, X. Yang and H. Zhang, Numerical approximations for Allen-Cahn type phase field model of two-phase incompressible fluids with moving contact lines, Commun. Comput. Phys. 21(3), 867-889 (2017).

[33] J. Shen, C. Wang, X. Wang and S.M.Wise, Second-order convex splitting schemes for gradient flows with Ehrlich-Schwoebel type energy: Application to thin film epitaxy, SIAM J. Numer. Anal. 50(1), 105-125 (2012).

[34] J. Shen and J. Xu, Convergence and error analysis for the scalar auxiliary variable (SAV) schemes to gradient flows, SIAM J. Numer. Anal. 56(5), 2895-2912 (2018).

[35] J. Shen, J. Xu and J. Yang, A new class of efficient and robust energy stable schemes for gradient flows, SIAM Rev. 61(3), 474-506 (2017).

[36] J. Shen, J. Xu and J. Yang, The scalar auxiliary variable (SAV) approach for gradient flows, 
J. Comput. Phys. 353, 407-416 (2018).

[37] J. Shen and X. Yang, Numerical approximations of Allen-Cahn and Cahn-Hilliard equations, Discrete Contin. Dyn. Syst. 28(4), 1669-1691 (2010).

[38] J. Shen and X. Yang, Energy stable schemes for Cahn-Hilliard phase-field model of two-phase incompressible flows, Chin. Ann. Math. Ser. B. 31(5), 743-758 (2010).

[39] M. Takenaka and T. Hashimoto, Early-stage spinodal decomposition in ternary blends composed of a random copolymer and homopolymers, Macromolecules 29(11), 4134-4141 (1996).

[40] Y. Tan, K. Xu, P. Wang, W. Li, S. Sun and L. Dong, High mechanical strength and rapid response rate of poly( $N$-isopropyl acrylamide) hydrogel crosslinked by starch-based nanospheres, Soft Matter. 6(7), 1467-1471 (2010).

[41] P. Vignal, L. Dalcin, D. Brown, N. Collier and V. Calo, An energy-stable convex splitting for the phase-field crystal equation, Comput. Struct. 158, 355-368 (2015).

[42] C. Wang, X. Wang and S.M. Wise, Unconditionally stable schemes for equations of thin film epitaxy, Discrete Contin. Dyn. Syst. 28(1), 405-423 (2010).

[43] C. Wang and S.M. Wise, An energy stable and convergent finite-difference scheme for the modified phase field crystal equation, SIAM J. Numer. Anal. 49(3), 945-969 (2011).

[44] L. Wang, Y. Huang and K. Jiang, Analysis of SAV finite element method to phase field crystal model, Numer. Math. Theory Methods Appl. 13(2), 372-399 (2020).

[45] Q. Wang, J.L. Mynar, M. Yoshida, E. Lee, M. Lee, K. Okuro, K. Kinbara and T. Aida, Highwater-content mouldable hydrogels by mixing clay and a dendritic molecular binder, Nature 463(7279), 339-343 (2010).

[46] X. Wang and H. Wang, Advances in the fabrication of hydrogels with well-defined structure and high mechanical strength, Chinese Polymer Bulletin 34(3), 1-6 (2018).

[47] S.M. Wise, C. Wang and J. Lowengrub, An energy-stable and convergent finite-difference scheme for the phase field crystal equation, SIAM J. Numer. Anal. 47(3), 2269-2288 (2009).

[48] X. Wu, G.J. van Zwieten and K.G. van der Zee, Stabilized second-order convex splitting schemes for Cahn-Hilliard models with application to diffuse-interface tumor-growth models, Int. J. Numer. Methods Biomed. Eng. 30(2), 180-203 (2014).

[49] C. Xu, C. Chen, X. Yang and X. He, Numerical approximations for the hydrodynamics coupled binary surfactant phase field model: Second-order, linear, unconditionally energy stable schemes, Commun. Comput. Phys. 17(3), 835-858 (2019).

[50] Z. Xu, X. Yang, H. Zhang and Z. Xie, Efficient and linear schemes for anisotropic Cahn-Hilliard model using the stabilized-Invariant Energy Quadratization (S-IEQ) approach, Comput. Phys. Commun. 238, 36-49 (2019).

[51] Z. Xu and H. Zhang, Stabilized semi-implicit numerical schemes for the Cahn-Hilliard-like equation with variable interfacial parameter, J. Comput. Appl. Math. 346, 307-322 (2018).

[52] Y. Yan, W. Chen, C. Wang and S.M. Wise, A second-order energy stable BDF numerical scheme for the Cahn-Hilliard equation, Commun. Comput. Phys. 23(2), 572-602 (2018).

[53] X. Yang, Linear, first and second-order, unconditionally energy stable numerical schemes for the phase field model of homopolymer blends, J. Comput. Phys. 327, 294-316 (2016).

[54] X. Yang, Numerical approximations for the Cahn-Hilliard phase field model of the binary fluidsurfactant system, J. Sci. Comput. 74(3), 1533-1553 (2018).

[55] X. Yang and L. Ju, Linear and unconditionally energy stable schemes for the binary fluidsurfactant phase field model, Comput. Methods Appl. Mech. Engrg. 318, 1005-1029 (2017).

[56] X. Yang and H. Yu, Efficient second order unconditionally stable schemes for a phase field moving contact line model using an invariant energy quadratization approch, SIAM J. Sci. Comput. 40(3), B889-B914 (2018).

[57] X. Yang and J. Zhao, Efficient linear schemes for the nonlocal Cahn-Hilliard equation of phase 
field models, Comput. Phys. Commun. 235, 234-245 (2019).

[58] X. Yang and J. Zhao, On linear and unconditionally energy stable algorithms for variable mobility Cahn-Hilliard type equation with logarithmic Flory-Huggins potential, Commun. Comput. Phys. 25(3) 703-728 (2019).

[59] X. Yang, J. Zhao and Q. Wang, Numerical approximations for the molecular beam epitaxial growth model based on the invariant energy quadratization method, J. Comput. Phys. 333, 104-127 (2017).

[60] X. Yang, J. Zhao, Q. Wang and J. Shen, Numerical approximations for a three-component CahnHilliard phase-field model based on the invariant energy quadratization method, Math. Models Methods Appl. Sci. 27(11), 1993-2030 (2017).

[61] D. Zhai and H. Zhang, Investigation on the application of the TDGL equation in macromolecular microsphere composite hydrogel, Soft Matter. 9(3), 820-825 (2012).

[62] C. Zhang, H. Li, X. Zhang and L. Ju, Linear and unconditionally energy stable schemes for the multi-component two-phase diffuse interface model with Peng-Robinson equation of state, Commun. Comput. Phys. 26(4), 1071-1097 (2019).

[63] J. Zhang, C. Chen, J. Wang and X. Yang, Efficient, second oder accurate, and unconditionally energy stable numerical scheme for a new hydrodynamics coupled binary phase-field surfactant system, Comput. Phys. Commun. 251, (2020).

[64] J. Zhang, C. Chen and X. Yang, Efficient and energy stable method for the Cahn-Hilliard phasefield model for diblock copolymers, Appl. Numer. Math. 151, 263-281 (2020).

[65] J. Zhang and X. Yang, A new magnetic-coupled Cahn-Hilliard phase-field model for diblock copolymers and its numerical approximations, Appl. Math. Lett. 107, (2020).

[66] J. Zhao, K. Jiao, J. Yang, C. He and H. Wang, Mechanically strong and thermosensitive macromolecular microsphere composite poly(N-isopropylacrylamide) hydrogels, Polymer. 54(6), 15961602 (2013).

[67] J. Zhao, Q. Wang and X. Yang, Numerical approximations for a phase field dendritic crystal growth model based on the invariant energy quadratization approach, Internat. J. Numer. Methods Engrg. 110(3), 279-300 (2017).

[68] G. Zhu, J. Kou, S. Sun, J. Yao and A. Li, Decoupled, energy stable schemes for a phase-field surfactant model, Commun. Comput. Phys. 2018, 67-77 (2018).

[69] J. Zhu, L. Chen, J. Shen and V. Tikare, Coarsening kinetics from a variable-mobility CahnHilliard equation: Application of a semi-implicit Fourier spectral method, Phys. Rev. E 60(4), 3564-3572 (1999). 